\title{
On the prescribed variable exponent mean curvature impulsive system boundary value problems
}

Li Yin ${ }^{1}$, Xiaopin Liu², Jingjing Liü ${ }^{2}$ and Qihu Zhang ${ }^{2 *}$

Dedicated to Professor Xianling Fan on his 70 birthday.

\section{"Correspondence:}

zhangqihu@yahoo.com

${ }^{2}$ College of Mathematics and

Information Science, Zhengzhou

University of Light Industry,

Zhengzhou, Henan 450002, China

Full list of author information is

available at the end of the article

\begin{abstract}
This paper investigates the existence of solutions for prescribed variable exponent mean curvature impulsive system, with periodic-like, Dirichlet, and Neumann boundary value conditions, respectively. The proof of our main result is based upon the Leray-Schauder degree. The sufficient conditions for the existence of solutions are given.

MSC: 34B37; 34B15
\end{abstract}

Keywords: variable exponent mean curvature; impulsive system; the Leray-Schauder degree

\section{Introduction}

In this paper, we consider the existence of solutions for the prescribed variable exponent mean curvature system

$$
-\left(\varphi\left(t, u^{\prime}\right)\right)^{\prime}+f\left(t, u, u^{\prime}\right)=0, \quad t \in(0, T), t \neq t_{i},
$$

where $u:[0, T] \rightarrow \mathbb{R}^{N}$, with the following impulsive conditions:

$$
\begin{aligned}
& \lim _{t \rightarrow t_{i}^{+}} u(t)-\lim _{t \rightarrow t_{i}^{-}} u(t)=A_{i}\left(\lim _{t \rightarrow t_{i}^{-}} u(t), \lim _{t \rightarrow t_{i}^{-}} u^{\prime}(t)\right), \quad i=1, \ldots, k, \\
& \lim _{t \rightarrow t_{i}^{+}} \varphi\left(t, u^{\prime}(t)\right)-\lim _{t \rightarrow t_{i}^{-}} \varphi\left(t, u^{\prime}(t)\right)=B_{i}\left(\lim _{t \rightarrow t_{i}^{-}} u(t), \lim _{t \rightarrow t_{i}^{-}} u^{\prime}(t)\right), \quad i=1, \ldots, k,
\end{aligned}
$$

and one of the following boundary value conditions:

$$
\begin{aligned}
& u(0)=u(T), \quad \text { and } \quad \varphi\left(0, u^{\prime}(0)\right)=\varphi\left(T, u^{\prime}(T)\right), \\
& u(0)=u(T)=0, \\
& u^{\prime}(0)=u^{\prime}(T)=0,
\end{aligned}
$$


where

$$
\varphi(t, x)=\frac{|x|^{p(t)-1} x}{\left(1+|x|^{q(t) p(t)}\right)^{\frac{1}{q(t)}}}, \quad \forall t \in[0, T], x \in \mathbb{R}^{N}
$$

$p, q \in C\left([0, T], \mathbb{R}^{+}\right)$are absolutely continuous; and $p, q$ satisfy $p(t) \geq 1, q(t) \geq 1 .-\left(\varphi\left(t, u^{\prime}\right)\right)^{\prime}$ is called the variable exponent mean curvature operator; $0<t_{1}<t_{2}<\cdots<t_{k}<T ; A_{i}, B_{i} \in$ $C\left(\mathbb{R}^{N} \times \mathbb{R}^{N}, \mathbb{R}^{N}\right)$.

If $p(0)=p(T)$ and $q(0)=q(T)$, then $\varphi\left(0, u^{\prime}(0)\right)=\varphi\left(T, u^{\prime}(T)\right)$ implies $u^{\prime}(0)=u^{\prime}(T)$, and (4) is the periodic boundary value condition. Thus we call (4) the periodic-like boundary value condition.

The system (1)-(3) is called a prescribed variable exponent mean curvature impulsive system. It has three characteristics, i.e. impulsive, mean curvature and variable exponent. Let us simply introduce the three characteristics.

The theory of impulsive differential equations describes processes which experience a sudden change of their state at certain moments, such as mechanical systems with impact, biological systems such as heart beats, blood flows, population dynamics, theoretical physics, radiophysics, pharmacokinetics, mathematical economy, chemical technology, electric technology, metallurgy, ecology, industrial robotics, biotechnology processes, chemistry, engineering, control theory, medicine, and so on. There are many results on the Laplacian impulsive differential equations boundary value problems (see [1-13]). The results as regards $p$-Laplacian impulsive differential equations boundary value problems are more difficult due to the nonlinearity of $p$-Laplacian (see [14-18]). Because of the impulsive conditions and the non-continuity of solutions, this paper has more difficulties than [19]. In many papers about the usual Laplacian impulsive problems, the authors use the impulsive condition $\lim _{t \rightarrow t_{i}^{+}} u^{\prime}(t)-\lim _{t \rightarrow t_{i}^{-}} u^{\prime}(t)=B_{i}\left(\lim _{t \rightarrow t_{i}^{-}} u(t), \lim _{t \rightarrow t_{i}^{-}} u^{\prime}(t)\right)$, but we think that the condition (3) is better in this paper. Therefore, we should consider what kind of impulsive condition is suitable for prescribed variable exponent mean curvature problems, it is a difference between this paper with the usual Laplacian impulsive problems.

Simultaneously, system (1) is a kind of mean curvature system. This kind of problems has attracted more and more attentions recently (see [20-28]). In [19], the authors generalized the usual mean curvature systems to variable exponent mean curvature systems, and discuss the existence of solutions of (1) with periodic-like boundary value condition (without impulsive conditions). In [29], the authors dealt with the existence of solutions and nonnegative solutions of (1)-(3) with initial boundary value condition. This paper deals with the existence of solutions of (1)-(3) with periodic-like boundary value condition, Neumann boundary value condition, or Dirichlet boundary value condition, respectively. This paper was motivated by [19, 29]. Similar to [19, 29], the proof of our main result is based upon the Leray-Schauder degree, but this paper is more difficult than $[19,29]$.

System (1) is also a kind of variable exponent equations. The variable exponent equations arise from the study of nonlinear elasticity, electrorheological fluids and image restoration, etc. We refer readers to [30-33] for detailed application backgrounds. There are many results on this kind of problems [30-48]. Many results show that problems with variable exponent growth conditions are more complex than those with constant exponent growth conditions. For instance we have the following. 
(a) If $\Omega \subset \mathbb{R}^{N}$ is a bounded domain, the infimum $\lambda_{p(\cdot)}$ of the eigenvalue of $p(x)$-Laplacian is zero in general, and the $p(x)$-Laplacian does not have the so-called first eigenfunction (see [39]); but the fact that $\lambda_{p}>0$ is very important in the study of $p$-Laplacian problems, and the first eigenfunction of the $p$-Laplacian was used to construct subsolutions of a $p$-Laplacian problem successfully (see [49]).

(b) For variable exponent function $L^{p(\cdot)}\left(\mathbb{R}^{N}\right)$, the norm $|\cdot|_{p(\cdot)}$ is not invariant for the translational coordinate transformation, i.e. under the usual Luxemburg norm $|\cdot|_{p(\cdot)}$, we have $|u(x)|_{p(\cdot)} \neq\left|u\left(x+x_{0}\right)\right|_{p(\cdot)}$ in general (see [50]).

(c) In [51], the author generalized the Picone identities for half-linear elliptic operators with $p(x)$-Laplacians and applications to Sturmian comparison theory, but the formula is different from the constant exponent case.

In this paper, we investigate the existence of solutions for the prescribed variable exponent mean curvature impulsive differential system boundary value problems, the proof of our main result is based upon the Leray-Schauder degree.

This paper is divided into four sections; in the second section, we will discuss the existence of solutions of variable exponent mean curvature impulsive system periodic-like boundary value problems. In the third section, we will discuss the existence of solutions of variable exponent mean curvature impulsive system Dirichlet boundary value problems. Finally, in the fourth section, we will discuss the existence of solutions of variable exponent mean curvature impulsive system Neumann boundary value problems.

\section{Periodic-like boundary value problems}

In this section, we will discuss the existence of solutions of variable exponent mean curvature impulsive system periodic-like boundary value problems, i.e. the existence of solutions of (1)-(4). In order to do that, we give the following notations and basic assumptions:

For any $v \in \mathbb{R}^{N}, v^{j}$ will denote the $j$ th component of $v$; the inner product in $\mathbb{R}^{N}$ will be denoted by $\langle\cdot, \cdot\rangle ;|\cdot|$ will denote the absolute value and the Euclidean norm on $\mathbb{R}^{N}$. Denote $J=[0, T], J^{\prime}=[0, T] \backslash\left\{t_{0}, t_{1}, \ldots, t_{k+1}\right\}, J_{0}=\left[t_{0}, t_{1}\right], J_{i}=\left(t_{i}, t_{i+1}\right], i=1, \ldots, k$, where $t_{0}=0, t_{k+1}=T$. Denote $J_{i}^{o}$ the interior of $J_{i}, i=0,1, \ldots, k$. Let $P C\left(J, \mathbb{R}^{N}\right)=\{x$ : $J \rightarrow \mathbb{R}^{N} \mid x \in C\left(J_{i}, \mathbb{R}^{N}\right), i=0,1, \ldots, k$, and $x\left(t_{i}^{+}\right)$exists for $\left.i=1, \ldots, k\right\} ; P C^{1}\left(J, \mathbb{R}^{N}\right)=\{x \in$ $P C\left(J, \mathbb{R}^{N}\right) \mid x^{\prime} \in C\left(J_{i}^{o}, \mathbb{R}^{N}\right), \lim _{t \rightarrow t_{i}^{+}} u^{\prime}(t)$ and $\lim _{t \rightarrow t_{i+1}^{-}} u^{\prime}(t)$ exist for $\left.i=0,1, \ldots, k\right\}$. For any $u(t)=\left(u^{1}(t), \ldots, u^{N}(t)\right) \in P C\left(J, \mathbb{R}^{N}\right)$, we denote $\left|u^{i}\right|_{0}=\sup \left\{\left|u^{i}(t)\right| \mid t \in J^{\prime}\right\}$. Obviously, $P C\left(J, \mathbb{R}^{N}\right)$ is a Banach space with the norm $\|u\|_{0}=\left(\sum_{i=1}^{N}\left|u^{i}\right|_{0}^{2}\right)^{\frac{1}{2}}, P C^{1}\left(J, \mathbb{R}^{N}\right)$ is a Banach space with the norm $\|u\|_{1}=\|u\|_{0}+\left\|u^{\prime}\right\|_{0}$. In the following, $P C\left(J, \mathbb{R}^{N}\right)$ and $P C^{1}\left(J, \mathbb{R}^{N}\right)$ will be simply denoted by $P C$ and $P C^{1}$, respectively. Denote $L^{1}=L^{1}\left(J, \mathbb{R}^{N}\right)$, and the norm in $L^{1}$ is $\|u\|_{L^{1}}=\left[\sum_{i=1}^{N}\left(\int_{0}^{T}\left|u^{i}(r)\right| d r\right)^{2}\right]^{\frac{1}{2}}$.

Let $N \geq 1$, the function $f: J \times \mathbb{R}^{N} \times \mathbb{R}^{N} \rightarrow \mathbb{R}^{N}$ is assumed to be Caratheodory, and by this we mean:

(i) for almost every $t \in J$ the function $f(t, \cdot, \cdot)$ is continuous;

(ii) for each $(x, y) \in \mathbb{R}^{N} \times \mathbb{R}^{N}$ the function $f(\cdot, x, y)$ is measurable on $J$;

(iii) for each $R>0$ there is a $\beta_{R} \in L^{1}(J, \mathbb{R})$ such that, for almost every $t \in J$ and every

$$
(x, y) \in \mathbb{R}^{N} \times \mathbb{R}^{N} \text { with }|x| \leq R,|y| \leq R \text {, one has }|f(t, x, y)| \leq \beta_{R}(t) .
$$

We say a function $u: J \rightarrow \mathbb{R}^{N}$ is a solution of (1) if $u \in P C^{1}$ with $\varphi\left(t, u^{\prime}\right)$ absolutely continuous on $J_{i}^{o}, i=0,1, \ldots, k$, which satisfies (1) a.e. on $J$.

In fact, the above notations and basic assumptions will be used in throughout the paper. 


\subsection{Preliminary}

In this subsection, we will make some preparations.

Lemma 2.1 (see [19]) $\varphi$ is a continuous function and satisfies the following.

(i) For any $t \in J, \varphi(t, \cdot)$ is strictly monotone, i.e.

$$
\left\langle\varphi\left(t, x_{1}\right)-\varphi\left(t, x_{2}\right), x_{1}-x_{2}\right\rangle>0, \quad \text { for any } x_{1}, x_{2} \in \mathbb{R}^{N}, x_{1} \neq x_{2} .
$$

(ii) For any fixed $t \in J, \varphi(t, \cdot)$ is a homeomorphism from $\mathbb{R}^{N}$ to

$$
E=\left\{x \in \mathbb{R}^{N}|| x \mid<1\right\} .
$$

For any $t \in J$, we denote by $\varphi^{-1}(t, \cdot)$ the inverse operator of $\varphi(t, \cdot)$, then

$$
\varphi^{-1}(t, x)=\left(1-|x|^{q(t)}\right)^{\frac{-1}{p(t) q(t)}}|x|^{\frac{1}{p(t)}-1} x, \quad \text { for } x \in E \backslash\{0\}, \varphi^{-1}(t, 0)=0 .
$$

Let us now consider the following simple problem:

$$
\left(\varphi\left(t, u^{\prime}(t)\right)\right)^{\prime}=g(t), \quad t \in(0, T), t \neq t_{i}
$$

with the following impulsive boundary value conditions:

$$
\left\{\begin{array}{l}
\lim _{t \rightarrow t_{i}^{+}} u(t)-\lim _{t \rightarrow t_{i}^{-}} u\left(t_{i}\right)=a_{i}, \quad i=1, \ldots, k, \\
\lim _{t \rightarrow t_{i}^{+}} \varphi\left(t, u^{\prime}(t)\right)-\lim _{t \rightarrow t_{i}^{-}} \varphi\left(t, u^{\prime}(t)\right)=b_{i}, \quad i=1, \ldots, k, \\
u(0)=u(T), \quad \varphi\left(0, u^{\prime}(0)\right)=\varphi\left(T, u^{\prime}(T)\right),
\end{array}\right.
$$

where $a_{i}, b_{i} \in \mathbb{R}^{N}, \sum_{i=1}^{k}\left|b_{i}\right|<1 ; g \in L^{1}$ and satisfies $\int_{0}^{T} g(t) d t+\sum_{i=1}^{k} b_{i}=0$.

Denote

$$
L_{m}^{1}=\left\{v \in L^{1} \mid \int_{0}^{T} v(r) d r=0\right\} .
$$

Let $h(t)=g(t)+\frac{1}{T} \sum_{i=1}^{k} b_{i}$, then $h \in L_{m}^{1}$. If $u$ is a solution of (7) with (8), by integrating (7) from 0 to $t$, we find that

$$
\varphi\left(t, u^{\prime}(t)\right)=\varphi\left(0, u^{\prime}(0)\right)+\sum_{t_{i}<t} b_{i}+\int_{0}^{t}\left[h(r)-\frac{1}{T} \sum_{i=1}^{k} b_{i}\right] d r, \quad \forall t \in J^{\prime} .
$$

Denote $\rho=\varphi\left(0, u^{\prime}(0)\right)$. Define operator $F: L^{1} \longrightarrow P C$ as

$$
F(g)(t)=\int_{0}^{t} g(t) d t, \quad \forall t \in J, \forall g \in L^{1}
$$

From (9) and (ii) in Lemma 2.1, we can see that

$$
\sup _{t \in J}\left|\varphi\left(t, u^{\prime}(t)\right)\right|=\sup _{t \in J}\left|\rho+\sum_{t_{i}<t} b_{i}+F\left(h-\frac{1}{T} \sum_{i=1}^{k} b_{i}\right)(t)\right|<1 .
$$


Denote

$$
\begin{aligned}
\widetilde{D}_{b}= & \left\{h \in L_{m}^{1} \mid \text { there exists } \rho \in \mathbb{R}^{N}\right. \text { such that } \\
& \left.\sup _{t \in J}\left|\rho+\sum_{t_{i}<t} b_{i}+F\left(h-\frac{1}{T} \sum_{i=1}^{k} b_{i}\right)(t)\right|<1\right\} .
\end{aligned}
$$

By (9), we have

$$
u(t)=u(0)+\sum_{t_{i}<t} a_{i}+F\left\{\varphi^{-1}\left[t,\left(\rho+\sum_{t_{i}<t} b_{i}+F\left(h-\frac{1}{T} \sum_{i=1}^{k} b_{i}\right)\right)\right]\right\}(t), \quad \forall t \in J,
$$

the boundary value conditions imply that

$$
\sum_{i=1}^{k} a_{i}+\int_{0}^{T} \varphi^{-1}\left\{t,\left[\rho+\sum_{t_{i}<t} b_{i}+F\left(h-\frac{1}{T} \sum_{i=1}^{k} b_{i}\right)(t)\right]\right\} d t=0
$$

Denote $a=\left(a_{1}, \ldots, a_{k}\right) \in \mathbb{R}^{k N}, b=\left(b_{1}, \ldots, b_{k}\right) \in \mathbb{R}^{k N}$. It is easy to see that $\rho$ is dependent on $a, b$ and $h$. For fixed $a, b \in \mathbb{R}^{k N}, h \in \widetilde{D}_{b}$, we define

$$
\Lambda_{(a, b, h)}(\rho)=\sum_{i=1}^{k} a_{i}+\int_{0}^{T} \varphi^{-1}\left\{t,\left[\rho+\sum_{t_{i}<t} b_{i}+F\left(h-\frac{1}{T} \sum_{i=1}^{k} b_{i}\right)(t)\right]\right\} d t
$$

Denote

$$
\begin{aligned}
D_{a, b}= & \left\{h \in L_{m}^{1} \mid \text { there exists } \rho \in \mathbb{R}^{N}\right. \text { such that } \\
& \left.\sup _{t \in J}\left|\rho+\sum_{t_{i}<t} b_{i}+F\left(h-\frac{1}{T} \sum_{i=1}^{k} b_{i}\right)(t)\right|<1 \text { and } \Lambda_{(a, b, h)}(\rho)=0\right\} .
\end{aligned}
$$

If (7) with (8) has a solution in $P C^{1}$, we must have $h=g(t)+\frac{1}{T} \sum_{i=1}^{k} b_{i} \in D_{a, b}$.

Denote $W_{m}=\mathbb{R}^{2 k N} \times L_{m}^{1}$ with the norm

$$
\|w\|_{W_{m}}=\sum_{i=1}^{k}\left|a_{i}\right|+\sum_{i=1}^{k}\left|b_{i}\right|+\|h\|_{L^{1}}, \quad \forall w=(a, b, h) \in W_{m}
$$

then $W_{m}$ is a Banach space.

Denote

$$
\begin{aligned}
& C_{*}=\frac{1}{6 N} \min _{t \in J}\left|\varphi^{-1}\left(t, \frac{1}{6 N}\right)\right|, \quad \mathcal{M}_{1}=\left\{\left(a_{1}, \ldots, a_{k}\right) \in \mathbb{R}^{k N}\left|\sum_{i=1}^{k}\right| a_{i} \mid<\frac{1}{4} T C_{*}\right\}, \\
& \mathcal{M}_{2}=\left\{\left(b_{1}, \ldots, b_{k}\right) \in \mathbb{R}^{k N}\left|\sum_{i=1}^{k}\right| b_{i} \mid<\frac{1}{12 N}\right\} .
\end{aligned}
$$

Lemma 2.2 The function $\Lambda_{w}(\cdot)$ has the following properties: 
$\left(1^{0}\right)$ For any fixed $(a, b) \in \mathcal{M}_{1} \times \mathcal{M}_{2}, \forall h \in D_{a, b}$ the equation

$$
\Lambda_{w}(\rho)=0, \quad \text { where } w=(a, b, h) \in W_{m},
$$

has a unique solution $\rho(w) \in \mathbb{R}^{N}$.

$\left(2^{0}\right)$ For any fixed $a=\left(a_{1}, \ldots, a_{k}\right) \in \mathcal{M}_{1}, b=\left(b_{1}, \ldots, b_{k}\right) \in \mathcal{M}_{2}, D_{a, b}$ contains the open ball $E\left(0, \frac{1}{12}\right):=\left\{u \in L_{m}^{1} \mid\|u\|_{L^{1}}<\frac{1}{12}\right\}$, and then one defines a mapping $\rho: \mathcal{M}_{1} \times \mathcal{M}_{2} \times$ $E\left(0, \frac{1}{12}\right) \rightarrow \mathbb{R}^{N}$.

$\left(3^{0}\right)$ The function $\rho: \mathcal{M}_{1} \times \mathcal{M}_{2} \times E\left(0, \frac{1}{12}\right) \rightarrow \mathbb{R}^{N}$, defined in $\left(2^{0}\right)$, is continuous and bounded.

$\left(4^{0}\right)$ For any fixed $a=\left(a_{1}, \ldots, a_{k}\right) \in \mathcal{M}_{1}, b=\left(b_{1}, \ldots, b_{k}\right) \in \mathcal{M}_{2}$, the set $D_{a, b}$ is open and unbounded in $L_{m}^{1}$.

Proof $\left(1^{0}\right)$ From the definition of $D_{a, b}$, for any fixed $(a, b) \in \mathcal{M}_{1} \times \mathcal{M}_{2}, \forall h \in D_{a, b}$, the equation $\Lambda_{w}(\rho)=0$ has at least one solution. Since $\varphi(t, \cdot)$ is strictly monotone, we can see that $\varphi^{-1}(t, \cdot)$ is strictly monotone. Thus, the equation $\Lambda_{w}(\rho)=0$ has a unique solution $\rho(w) \in \mathbb{R}^{N}$.

$\left(2^{0}\right)$ Let $h \in E\left(0, \frac{1}{12}\right)$, then $0 \leq\|h\|_{L^{1}}<\frac{1}{12}$. Denote

$$
g=h-\frac{1}{T} \sum_{i=1}^{k} b_{i}, \quad A=\left\{x \in \mathbb{R}^{N}|| x \mid<\frac{2}{3}\right\}
$$

Obviously, for any $(a, b, h) \in \mathcal{M}_{1} \times \mathcal{M}_{2} \times E\left(0, \frac{1}{12}\right)$, for any $\rho \in \bar{A}, \Lambda_{(a, b, h)}(\rho)$ is well defined, i.e.,

$$
\sup _{t \in J}\left|\rho+\sum_{t_{i}<t} b_{i}+F(g)(t)\right|<1 \text {. }
$$

Observe that $0 \leq\left|\sum_{t_{i}<t} b_{i}+F(g)(t)\right|<\frac{1}{4}$ and $\frac{5}{12}<\left|\rho+\sum_{t_{i}<t} b_{i}+F(g)(t)\right|<\frac{11}{12}$ for any $(t, \rho) \in J \times \partial A$.

Since $\left|\rho+\sum_{t_{i}<t} b_{i}+F(g)(t)\right|-\left|\sum_{t_{i}<t} b_{i}+F(g)(t)\right|>\frac{1}{6}$ for any $(t, \rho) \in J \times \partial A$, we have

$$
\begin{aligned}
\left\langle\Lambda_{w}(\rho), \rho\right\rangle= & \left\langle\sum_{i=1}^{k} a_{i}, \rho\right\rangle+\int_{0}^{T}\left\langle\varphi^{-1}\left\{t,\left[\rho+\sum_{t_{i}<t} b_{i}+F(g)(t)\right]\right\}, \rho+\sum_{t_{i}<t} b_{i}+F(g)(t)\right\rangle d t \\
& -\int_{0}^{T}\left\langle\varphi^{-1}\left\{t,\left[\rho+\sum_{t_{i}<t} b_{i}+F(g)(t)\right]\right\}, \sum_{t_{i}<t} b_{i}+F(g)(t)\right\rangle d t \\
\geq & \int_{0}^{T}\left(1-\left|\rho+\sum_{t_{i}<t} b_{i}+F(g)(t)\right|^{q(t)}\right)^{\frac{-1}{p(t) q(t)}} \\
& \times\left|\rho+\sum_{t_{i}<t} b_{i}+F(g)(t)\right|^{\frac{1}{p(t)}+1} d t-\frac{2}{3} \sum_{i=1}^{k}\left|a_{i}\right| \\
& -\int_{0}^{T}\left(1-\left|\rho+\sum_{t_{i}<t} b_{i}+F(g)(t)\right|^{q(t)}\right)^{\frac{-1}{p(t) q(t)}}
\end{aligned}
$$




$$
\begin{aligned}
& \times\left|\rho+\sum_{t_{i}<t} b_{i}+F(g)(t)\right|^{\frac{1}{p(t)}}\left|\sum_{t_{i}<t} b_{i}+F(g)(t)\right| d t \\
= & \int_{0}^{T}\left(1-\left|\rho+\sum_{t_{i}<t} b_{i}+F(g)(t)\right|^{q(t)}\right)^{\frac{-1}{p(t) q(t)}}\left|\rho+\sum_{t_{i}<t} b_{i}+F(g)(t)\right|^{\frac{1}{p(t)}} \\
& \times\left(\left|\rho+\sum_{t_{i}<t} b_{i}+F(g)(t)\right|-\left|\sum_{t_{i}<t} b_{i}+F(g)(t)\right|\right) d t-\frac{2}{3} \sum_{i=1}^{k}\left|a_{i}\right| \\
\geq & C_{*} \int_{0}^{T}\left(\left|\rho+\sum_{t_{i}<t} b_{i}+F(g)(t)\right|-\left|\sum_{t_{i}<t} b_{i}+F(g)(t)\right|\right) d t-\frac{2}{3} \sum_{i=1}^{k}\left|a_{i}\right| \\
\geq & \frac{1}{6} T C_{*}-\frac{2}{3} \sum_{i=1}^{k}\left|a_{i}\right|>0 .
\end{aligned}
$$

This means that $\left\langle\Lambda_{w}(\rho), \rho\right\rangle>0$ for any $\rho \in \partial A$.

For any fixed $(a, b, x) \in \mathcal{M}_{1} \times \mathcal{M}_{2} \times E\left(0, \frac{1}{12}\right)$, let us consider

$$
\Gamma_{1}(\rho, \lambda)=\lambda \Lambda_{(a, b, x)}(\rho)+(1-\lambda) \rho .
$$

It is easy to see that $\Gamma_{1}(\rho, \lambda)=0$ has no solution on $\partial A$ for any $\lambda \in[0,1]$. According to the homotopy invariance property of the Brouwer degree, we can see that $\Lambda_{w}(\rho)=0$ possesses a solution in $A$. Thus $E\left(0, \frac{1}{12}\right) \subset D_{a, b}$, and one then defines a mapping $\rho: \mathcal{M}_{1} \times$ $\mathcal{M}_{2} \times E\left(0, \frac{1}{12}\right) \rightarrow \mathbb{R}^{N}$.

$\left(3^{0}\right)$ For any $(a, b, h) \in \mathcal{M}_{1} \times \mathcal{M}_{2} \times E\left(0, \frac{1}{12}\right)$, from the definition of $D_{a, b}$, we have

$$
\sup _{t \in J}\left|\rho+\sum_{t_{i}<t} b_{i}+F\left(h-\frac{1}{T} \sum_{i=1}^{k} b_{i}\right)(t)\right|<1 .
$$

Since $\sum_{t_{i}<0} b_{i}+F\left(h-\frac{1}{T} \sum_{i=1}^{k} b_{i}\right)(0)=0$, we have

$$
|\rho|=\left|\rho+\sum_{t_{i}<0} b_{i}+F\left(h-\frac{1}{T} \sum_{i=1}^{k} b_{i}\right)(0)\right|<1 .
$$

Thus the mapping $\rho$ is bounded.

Now, let us prove the continuity of $\rho$. Let $w_{n}=\left(a_{n}, b_{n}, h_{n}\right) \in \mathcal{M}_{1} \times \mathcal{M}_{2} \times E\left(0, \frac{1}{12}\right)$ is a convergent sequence in $\mathcal{M}_{1} \times \mathcal{M}_{2} \times E\left(0, \frac{1}{12}\right)$, and $w_{n} \rightarrow w_{0}=\left(a_{0}, b_{0}, h_{0}\right) \in \mathcal{M}_{1} \times \mathcal{M}_{2} \times$ $E\left(0, \frac{1}{12}\right)$ as $n \rightarrow+\infty$, where $b_{0}=\left(b_{1,0}, \ldots, b_{k, 0}\right)$. Since $\left\{\rho\left(w_{n}\right)\right\}$ is a bounded sequence, it contains a convergent subsequence $\left\{\rho\left(w_{n_{j}}\right)\right\}$. We may assume that $\rho\left(w_{n_{j}}\right) \rightarrow \rho_{0}$ as $j \rightarrow+\infty$.

If $\Lambda_{w_{0}}\left(\rho_{0}\right)$ is well defined, i.e., $\sup _{t \in J}\left|\rho_{0}+\sum_{t_{i}<t} b_{i, 0}+F\left(h_{0}-\frac{1}{T} \sum_{i=1}^{k} b_{i, 0}\right)(t)\right|<1$. Since $\Lambda_{w_{n_{j}}}\left(\rho\left(w_{n_{j}}\right)\right)=0$, letting $j \rightarrow+\infty$, we have $\Lambda_{w_{0}}\left(\rho_{0}\right)=0$. Combining with $\left(1^{0}\right)$, we get $\rho_{0}=$ $\rho\left(w_{0}\right)$. This means that $\rho$ is continuous.

It only remains to prove that $\Lambda_{w_{0}}\left(\rho_{0}\right)$ is well defined.

Denote by $E^{*} \subset \mathbb{R}^{N}$ an open ball which centered at $\rho\left(w_{0}\right)$ such that $\Lambda_{w_{0}}(\cdot)$ is well defined on $\overline{E^{*}}$. According to Lemma 2.1, we have

$$
\left\langle\Lambda_{w_{0}}(\rho), \rho-\rho\left(w_{0}\right)\right\rangle=\left\langle\Lambda_{w_{0}}(\rho)-\Lambda_{w_{0}}\left(\rho\left(w_{0}\right)\right), \rho-\rho\left(w_{0}\right)\right\rangle \geq 0, \quad \forall \rho \in \overline{E^{*}},
$$




$$
\left\langle\Lambda_{w_{0}}(\rho), \rho-\rho\left(w_{0}\right)\right\rangle>0, \quad \forall \rho \in \partial \overline{E^{*}} .
$$

It is easy to see that there exists a neighborhood $U \subset E\left(0, \frac{1}{12}\right)$ of $h_{0}$ in $L_{m}^{1}, U_{1} \subset \mathcal{M}_{1}$ is a neighborhood of $a_{0}, U_{2} \subset \mathcal{M}_{2}$ is a neighborhood of $b_{0}$, such that, for each $(a, b, y) \in$ $U_{1} \times U_{2} \times U, \Lambda_{(a, b, y)}(\cdot)$ is well defined on $\overline{E^{*}}$. In addition, the mapping

$$
(a, b, y) \longmapsto \inf _{\rho \in \partial \bar{E}^{*}}\left\langle\Lambda_{(a, b, y)}(\rho), \rho-\rho\left(w_{0}\right)\right\rangle
$$

is easily seen to be continuous in $U_{1} \times U_{2} \times U$. Thus, there exists a neighborhood $V_{1} \times$ $V_{2} \times V \subset U_{1} \times U_{2} \times U$ of $w_{0}$ such that

$$
\left\langle\Lambda_{(a, b, x)}(\rho), \rho-\rho\left(w_{0}\right)\right\rangle>0, \quad \forall \rho \in \partial \overline{E^{*}}, \forall(a, b, x) \in V_{1} \times V_{2} \times V .
$$

For any fixed $(a, b, x) \in V_{1} \times V_{2} \times V$, let us consider

$$
\Gamma_{2}(\rho, \lambda)=\lambda \Lambda_{(a, b, x)}(\rho)+(1-\lambda)\left(\rho-\rho\left(w_{0}\right)\right) .
$$

Obviously, $\Gamma_{2}(\rho, \lambda)=0$ has no solution on $\partial \overline{E^{*}}$ for any $\lambda \in[0,1]$.

For any $(a, b, x) \in V_{1} \times V_{2} \times V$, according to the homotopy invariance property of the Brouwer degree, we conclude that the equation $\Lambda_{(a, b, x)}(\rho)=0$ has its (unique) solution on $\overline{E^{*}}$. Since $w_{n_{j}} \rightarrow w_{0}$ as $j \rightarrow+\infty$, we have $w_{n_{j}} \in V_{1} \times V_{2} \times V$ when $j$ is large enough, and $\rho\left(w_{n_{j}}\right) \in \overline{E^{*}}$. Since $\rho\left(w_{n_{j}}\right) \rightarrow \rho_{0}$ as $j \rightarrow+\infty, \rho_{0} \in \overline{E^{*}}$. This means that $\Lambda_{w_{0}}\left(\rho_{0}\right)$ is well defined.

$\left(4^{0}\right)$ Let $u_{n}(t)=\frac{n}{N T} \sin \frac{4 n \pi t}{T} \cdot(1,0, \ldots, 0)$, then $u_{n} \in L_{m}^{1}$. Denote $A_{0}=\left\{x \in \mathbb{R}^{N}|| x \mid<\frac{1}{2}\right\}$. For any $n \in N^{+}, \forall a \in \mathcal{M}_{1}, \forall b \in \mathcal{M}_{2}$ and $\forall \rho \in A_{0}$, we can see the following function is well defined:

$$
\Lambda_{n}(\rho)=\sum_{i=1}^{k} a_{i}+\int_{0}^{T} \varphi^{-1}\left\{t,\left[\rho+\sum_{t_{i}<t} b_{i}+F\left(u_{n}-\frac{1}{T} \sum_{i=1}^{k} b_{i}\right)(t)\right]\right\} d t .
$$

If $|\rho|=\frac{1}{2}$, then there exists some $\rho^{j}$ such that $\left|\rho^{j}\right| \geq \frac{1}{2 N}$. Without loss of generality, we may assume that $\rho^{j} \geq \frac{1}{2 N}$. Since $\sup _{t \in J}\left|F\left(u_{n}\right)(t)\right| \leq \frac{1}{2 N \pi}$ and $\sum_{i=1}^{k}\left|b_{i}\right|<\frac{1}{12 N}$, we have

$$
\left|\sum_{t_{i}<t} b_{i}+F\left(u_{n}-\frac{1}{T} \sum_{i=1}^{k} b_{i}\right)(t)\right|<\frac{1}{12 N}+\frac{1}{2 N \pi}+\frac{1}{12 N}<\frac{1}{3 N}, \quad \forall t \in J .
$$

Denote $z_{n}(t)=\rho+\sum_{t_{i}<t} b_{i}+F\left(u_{n}-\frac{1}{T} \sum_{i=1}^{k} b_{i}\right)$. Obviously, $\frac{1}{6}<\left|z_{n}(t)\right|<\frac{5}{6}, \forall t \in J$.

Since $\rho^{j} \geq \frac{1}{2 N}$, the $j$ th component $z_{n}^{j}$ of $z_{n}$ satisfies $z_{n}^{j}(t)>\frac{1}{6 N}, \forall t \in J$. Notice that $p(t) \geq 1$, then we can see that the $j$ th component $\left(\varphi^{-1}\left(t, z_{n}(t)\right)\right)^{j}$ of $\varphi^{-1}\left(t, z_{n}(t)\right)$ satisfies

$$
\begin{aligned}
\left(\varphi^{-1}\left(t, z_{n}(t)\right)\right)^{j} & =\left(1-\left|z_{n}(t)\right|^{q(t)}\right)^{\frac{-1}{p(t) q(t)}}\left|z_{n}(t)\right|^{\frac{1}{p(t)}-1} z_{n}^{j}(t) \\
& \geq\left(1-\left|\frac{1}{6 N}\right|^{q(t)}\right)^{\frac{-1}{p(t) q(t)}}\left|\frac{1}{6 N}\right|^{\frac{1}{p(t)}-1} \frac{1}{6 N} \geq C_{*} .
\end{aligned}
$$

Consider $\Phi(\rho, \lambda)=\lambda \Lambda_{n}(\rho)+(1-\lambda) \rho$. Since $C_{*}=\frac{1}{6 N} \min _{t \in J} \varphi^{-1}\left(t, \frac{1}{6 N}\right)$ and $\sum_{i=1}^{k}\left|a_{i}\right|<$ $\frac{1}{4} T C_{*}$, we have $\Lambda_{n}^{j}(\rho)>T C_{*}-\sum_{i=1}^{k}\left|a_{i}\right|>0$. Hence $\Lambda_{n}^{j}(\rho)$ and $\rho^{j}$ have the same sign, then 
$\Phi(\rho, \lambda)$ has no solution on $\partial A_{0}$ for any $\lambda \in[0,1]$. Thus

$$
d_{B}\left(\Phi(\rho, 1), A_{0}, 0\right)=d_{B}\left(\Phi(\rho, 0), A_{0}, 0\right)=1 .
$$

Thus $0=\Phi(\rho, 1)=\Lambda_{n}(\rho)$ has a solution on $\overline{A_{0}}$. Therefore $\left\{u_{n}\right\} \subset D_{a, b}$, This means that $D_{a, b}$ is unbounded.

Similar to the proof of $\left(3^{0}\right)$, for any $w_{0}=\left(a, b, h_{0}\right) \in\{(a, b)\} \times D_{a, b}$, there exists a neighborhood $V \subseteq U$ of $h_{0}$, and a neighborhood $E^{*} \subset \mathbb{R}^{N}$ of $\rho\left(w_{0}\right)$ such that $\Lambda_{(a, b, x)}(\rho)$ is well defined for any $(a, b, x) \in\{(a, b)\} \times V$ and $\rho \in \overline{E^{*}}$, which satisfies the requirement that, for any $(a, b, x) \in\{(a, b)\} \times V, \Lambda_{(a, b, x)}(\rho)=0$ has a solution $\rho \in \overline{E^{*}}$. This means that the set $D_{a, b}$ is open in $L_{m}^{1}$.

This completes the proof.

We continue now with our argument previous to Lemma 2.2. Let us define

$$
\begin{aligned}
& P: P C^{1} \rightarrow P C^{1}, \quad u \longmapsto u(0) ; \quad Q: L^{1} \rightarrow L^{1}, \quad h \longmapsto \frac{1}{T} \int_{0}^{T} h(t) d t ; \\
& \Theta_{b}: L^{1} \rightarrow L^{1}, \quad h \longmapsto(I-Q) h-\frac{1}{T} \sum_{i=1}^{k} b_{i}, \quad \text { for any fixed } b \in \mathbb{R}^{N} .
\end{aligned}
$$

We can split $L^{1}$ as $L^{1}=L_{m}^{1}+\mathcal{F}_{2}$, where $\mathcal{F}_{2}$ is the $N$-dimensional subspace of constant mappings. The operator $Q$ is a continuous projection from $L^{1}$ onto $\mathcal{F}_{2}$. Let us consider the subset $\widehat{D}_{a, b}$ of $L^{1}$ which is given by

$$
\widehat{D}_{a, b}=D_{a, b}+\mathcal{F}_{2}
$$

and define the nonlinear operator $K_{(a, b)}: \widehat{D}_{a, b} \rightarrow P C^{1}$, as

$$
K_{(a, b)}(h)(t)=F\left\{\varphi^{-1}\left[t,\left(\rho+\sum_{t_{i}<t} b_{i}+F(h)\right)\right]\right\}(t), \quad \forall t \in J .
$$

We say $\bar{U}$ is a closed equi-integrable set in $\widehat{D}_{a, b}$, if there exists $\beta \in L^{1}$, such that, for any $u \in \bar{U}$

$$
|u(t)| \leq \beta(t) \quad \text { a.e. on } J .
$$

Lemma 2.3 If $(a, b) \in \mathcal{M}_{1} \times \mathcal{M}_{2}$, then the operator $\left(K_{(a, b)} \circ \Theta_{b}\right)(\cdot)$ is continuous and sends closed equi-integrable subsets of $\widehat{D}_{a, b}$ into relatively compact sets in $P C^{1}$.

Proof It is easy to check that $\left(K_{(a, b)} \circ \Theta_{b}\right)(h)(\cdot) \in P C^{1}, \forall h \in \widehat{D}_{a, b}$. Since

$$
\left(K_{(a, b)} \circ \Theta_{b}\right)(h)^{\prime}(t)=\varphi^{-1}\left\{t,\left[\rho+\sum_{t_{i}<t} b_{i}+F\left(\Theta_{b}(h)\right)\right]\right\}, \quad \forall t \in J
$$

it is easy to check that $\left(K_{(a, b)} \circ \Theta_{b}\right)(\cdot)$ is a continuous operator from $\widehat{D}_{a, b}$ to $P C^{1}$. 
Let now $\bar{U}$ be a closed equi-integrable set in $\widehat{D}_{a, b}$, then there exists $\beta \in L^{1}$, such that, for any $u \in \bar{U}$,

$$
|u(t)| \leq \beta(t) \quad \text { a.e. on } J .
$$

We want to show that $\left(K_{(a, b)} \circ \Theta_{b}\right)(\bar{U}) \subset P C^{1}$ is a compact set.

Let $\left\{u_{n}\right\}$ is a sequence in $\left(K_{(a, b)} \circ \Theta_{b}\right)(\bar{U})$, then there exists a sequence $\left\{h_{n}\right\} \in \bar{U}$ such that $u_{n}=\left(K_{(a, b)} \circ \Theta_{b}\right)\left(h_{n}\right)$. For any $r_{1}, r_{2} \in J$, we have

$$
\left|F\left(\Theta_{b}\left(h_{n}\right)\right)\left(r_{1}\right)-F\left(\Theta_{b}\left(h_{n}\right)\right)\left(r_{2}\right)\right| \leq\left|\int_{r_{1}}^{r_{2}} \beta(t) d t\right|+\left|r_{1}-r_{2}\right| \frac{1}{T}\left(\int_{0}^{T} \beta(t) d t+\left|\sum_{i=1}^{k} b_{i}\right|\right) .
$$

Hence the sequence $\left\{F\left(\Theta_{b}\left(h_{n}\right)\right)\right\}$ is uniformly bounded and equi-continuous. By the Ascoli-Arzela theorem, there exists a subsequence of $\left\{F\left(\Theta_{b}\left(h_{n}\right)\right)\right\}$ (which we rename the same) is convergent in $P C$. According to the bounded continuity of the operator $\rho$, we can choose a subsequence of $\left\{\rho\left(a, b, \Theta_{b}\left(h_{n}\right)\right)+F\left(\Theta_{b}\left(h_{n}\right)\right)\right\}$ (which we still denote $\left.\left\{\rho\left(a, b, \Theta_{b}\left(h_{n}\right)\right)+F\left(\Theta_{b}\left(h_{n}\right)\right)\right\}\right)$ which is convergent in $P C$, then the sequence

$$
\varphi\left(t,\left(K_{(a, b)} \circ \Theta_{b}\right)\left(h_{n}\right)^{\prime}(t)\right)=\rho+\sum_{t_{i}<t} b_{i}+F\left(\Theta_{b}\left(h_{n}\right)\right)
$$

is convergent according to the norm in $P C$, by which, combined with the continuous of $\varphi^{-1}$, we can see

$$
\left(K_{(a, b)} \circ \Theta_{b}\right)\left(h_{n}\right)^{\prime}(t)=\varphi^{-1}\left[t,\left(\rho+\sum_{t_{i}<t} b_{i}+F\left(\Theta_{b}\left(h_{n}\right)\right)\right)\right](t), \quad \forall t \in J,
$$

is convergent according to the norm in $P C$.

Since

$$
\left(K_{(a, b)} \circ \Theta_{b}\right)\left(h_{n}\right)(t)=F\left\{\varphi^{-1}\left[t,\left(\rho+\sum_{t_{i}<t} b_{i}+F\left(\Theta_{b}\left(h_{n}\right)\right)\right)\right]\right\}(t), \quad \forall t \in J,
$$

according to the continuity of $\varphi^{-1}$, we can see $\left(K_{(a, b)} \circ \Theta_{b}\right)\left(h_{n}\right)$ is convergent in $P C$. Thus we conclude that $\left\{u_{n}\right\}$ is convergent in $P C^{1}$. This completes the proof.

We denote by $N_{f}(u): P C^{1} \rightarrow L^{1}$ the Nemytskii operator associated to $f$ defined by

$$
N_{f}(u)(t)=f\left(t, u(t), u^{\prime}(t)\right), \quad \text { a.e. on } J
$$

Denote

$$
\begin{aligned}
& \Theta_{\#}\left(N_{f}\right)(u)=(I-Q) N_{f}(u)-\frac{1}{T} \sum_{i=1}^{k} B_{i}, \\
& K(u)=F\left\{\varphi^{-1}\left[t,\left(\rho+\sum_{t_{i}<t} B_{i}+F\left(\Theta_{\#}\left(N_{f}\right)(u)\right)\right)\right]\right\}(t),
\end{aligned}
$$


and

$$
\Lambda_{u}^{*}(\rho)=\sum_{i=1}^{k} A_{i}+\int_{0}^{T} \varphi^{-1}\left\{t,\left[\rho+\sum_{t_{i}<t} B_{i}+\int_{0}^{t} f\left(r, u, u^{\prime}\right) d r\right]\right\} d t
$$

where $A_{i}=A_{i}\left(\lim _{t \rightarrow t_{i}^{-}} u(t), \lim _{t \rightarrow t_{i}^{-}} u^{\prime}(t)\right), B_{i}=B_{i}\left(\lim _{t \rightarrow t_{i}^{-}} u(t), \lim _{t \rightarrow t_{i}^{-}} u^{\prime}(t)\right), \rho$ is a solution of $\Lambda_{u}^{*}(\rho)=0$.

We assume

$\left(\mathrm{H}_{1}\right) \sum_{i=1}^{k}\left|A_{i}(x, y)\right|<\frac{1}{4} T C_{*}$ and $\sum_{i=1}^{k}\left|B_{i}(x, y)\right|<\frac{1}{12 N}, \forall(x, y) \in \mathbb{R}^{2 N}$.

Lemma 2.4 If $\left(\mathrm{H}_{1}\right)$ is satisfied, then $u$ is a solution of (1)-(4) if and only if $u$ is a solution of the following abstract operator equation:

$$
u=P u+\sum_{t_{i}<t} A_{i}+\frac{1}{T} \sum_{i=1}^{k} B_{i}+Q N_{f}(u)+K(u) .
$$

Proof (i) If $u$ is a solution of (1)-(4), by integrating (1) from 0 to $t$, we find that

$$
\varphi\left(t, u^{\prime}(t)\right)=\rho+\sum_{t_{i}<t} B_{i}+\int_{0}^{t} f\left(t, u, u^{\prime}\right) d t .
$$

From (4), we have

$$
\frac{1}{T} \sum_{i=1}^{k} B_{i}+\frac{1}{T} \int_{0}^{T} f\left(t, u, u^{\prime}\right) d t=\frac{1}{T} \sum_{i=1}^{k} B_{i}+Q N_{f}(u)=0,
$$

thus $\Theta_{\#}\left(N_{f}\right)(u)=N_{f}(u)$.

From (14), we have

$$
u=P u+\sum_{t_{i}<t} A_{i}+\frac{1}{T} \sum_{i=1}^{k} B_{i}+Q N_{f}(u)+K(u),
$$

where $\rho$ satisfies

$$
\sum_{i=1}^{k} A_{i}+F\left\{\varphi^{-1}\left[t,\left(\rho+\sum_{t_{i}<t} B_{i}+F\left(\Theta_{\#}\left(N_{f}\right)(u)\right)\right)\right]\right\}(T)=0 .
$$

Hence $u$ is a solution of (13).

(ii) If $u$ is a solution of (13), it is easy to see that (2) is satisfied. Let $t=0$, we have

$$
u(0)=P u+\frac{1}{T} \sum_{i=1}^{k} B_{i}+Q N_{f}(u)
$$

then

$$
\frac{1}{T} \sum_{i=1}^{k} B_{i}+Q N_{f}(u)=0
$$

thus $\Theta_{\#}\left(N_{f}\right)(u)=N_{f}(u)$. 
By the definition of the mapping $\rho$, we have

$$
\sum_{i=1}^{k} A_{i}+F\left\{\varphi^{-1}\left[t,\left(\rho+\sum_{t_{i}<t} B_{i}+F\left(\Theta_{\#}\left(N_{f}\right)(u)\right)\right)\right]\right\}(T)=0
$$

then $u(0)=u(T)$. From (13) and (15), we also have

$$
\begin{aligned}
& \varphi\left(t, u^{\prime}\right)=\rho+\sum_{t_{i}<t} B_{i}+F\left(\Theta_{\#}\left(N_{f}\right)(u)\right)(t), \quad t \in(0, T), t \neq t_{i}, \\
& \left(\varphi\left(t, u^{\prime}\right)\right)^{\prime}=f\left(t, u, u^{\prime}\right), \quad t \in(0, T), t \neq t_{i} .
\end{aligned}
$$

From (16), we find that (3) is satisfied. Since $\sum_{i=1}^{k} B_{i}+F\left(\Theta_{\#}\left(N_{f}\right)(u)\right)(T)=0$, we have

$$
\varphi\left(0, u^{\prime}(0)\right)=\varphi\left(T, u^{\prime}(T)\right)
$$

Hence $u$ is a solution of (1)-(4). This completes the proof.

\subsection{Existence of solutions}

In this subsection, we will apply the Leray-Schauder degree to deal with the existence of solutions of (1)-(4).

Theorem 2.5 If $\left(\mathrm{H}_{1}\right)$ is satisfied, $\Omega \subset P C^{1}$ is open bounded such that the following conditions hold:

$\left(1^{0}\right)$ For any $u \in \bar{\Omega}$, the mapping $t \rightarrow f\left(t, u, u^{\prime}\right)$ belongs to $\left\{u \in L^{1} \mid\|u\|_{L^{1}}<\frac{1}{24}\right\}$.

$\left(2^{0}\right)$ For each $\lambda \in(0,1)$, the problem

$$
\left\{\begin{array}{l}
\left(\varphi\left(t, u^{\prime}\right)\right)^{\prime}=\lambda f\left(t, u, u^{\prime}\right), \quad t \in(0, T), t \neq t_{i}, \\
\quad \lim _{t \rightarrow t_{i}^{+}} u(t)-\lim _{t \rightarrow t_{i}^{-}} u(t) \\
\quad=\lambda A_{i}\left(\lim _{t \rightarrow t_{i}^{-}} u(t), \lim _{t \rightarrow t_{i}^{-}} u^{\prime}(t)\right), \quad i=1, \ldots, k, \\
\lim _{t \rightarrow t_{i}^{+}} \varphi\left(t, u^{\prime}(t)\right)-\lim _{t \rightarrow t_{i}^{-}} \varphi\left(t, u^{\prime}(t)\right) \\
\quad=\lambda B_{i}\left(\lim _{t \rightarrow t_{i}^{-}} u(t), \lim _{t \rightarrow t_{i}^{-}} u^{\prime}(t)\right), \quad i=1, \ldots, k, \\
u(0)=u(T), \quad \text { and } \varphi\left(0, u^{\prime}(0)\right)=\varphi\left(T, u^{\prime}(T)\right)
\end{array}\right.
$$

has no solution on $\partial \Omega$.

$\left(3^{0}\right)$ The equation

$$
\omega(l):=\frac{1}{T} \int_{0}^{T} f(t, l, 0) d t+\frac{1}{T} \sum_{i=1}^{k} B_{i}(l, 0)=0
$$

has no solution on $\partial \Omega \cap \mathcal{F}_{2}$.

$\left(4^{0}\right)$ The Brouwer degree $d_{B}\left[\omega, \Omega \cap \mathcal{F}_{2}, 0\right] \neq 0$.

Then (1)-(4) has a solution on $\bar{\Omega}$.

Proof Denote

$$
A_{i}=A_{i}\left(\lim _{t \rightarrow t_{i}^{-}} u(t), \lim _{t \rightarrow t_{i}^{-}} u^{\prime}(t)\right), \quad B_{i}=B_{i}\left(\lim _{t \rightarrow t_{i}^{-}} u(t), \lim _{t \rightarrow t_{i}^{-}} u^{\prime}(t)\right) .
$$


Let us consider the following impulsive equation:

$$
\left\{\begin{array}{l}
\left(\varphi\left(t, u^{\prime}\right)\right)^{\prime}=\lambda N_{f}(u)+(1-\lambda)\left[Q N_{f}(u)+\frac{1}{T} \sum_{i=1}^{k} B_{i}\right], \quad t \in(0, T), t \neq t_{i}, \\
\lim _{t \rightarrow t_{i}^{+}} u(t)-\lim _{t \rightarrow t_{i}^{-}} u(t)=\lambda A_{i}, \quad i=1, \ldots, k \\
\lim _{t \rightarrow t_{i}^{+}} \varphi\left(t, u^{\prime}(t)\right)-\lim _{t \rightarrow t_{i}^{-}} \varphi\left(t, u^{\prime}(t)\right)=\lambda B_{i}, \quad i=1, \ldots, k \\
u(0)=u(T), \quad \varphi\left(0, u^{\prime}(0)\right)=\varphi\left(T, u^{\prime}(T)\right)
\end{array}\right.
$$

For any $\lambda \in(0,1]$, observe that, if $u$ is a solution to (17) or $u$ is a solution to (20), we have necessarily

$$
Q N_{f}(u)+\frac{1}{T} \sum_{i=1}^{k} B_{i}=0
$$

This means that (17) and (20) have the same solutions for $\lambda \in(0,1]$.

We denote $N(\cdot, \cdot): P C^{1} \times[0,1] \rightarrow L^{1}$ defined by

$$
N(u, \lambda)=\lambda N_{f}(u)+(1-\lambda)\left[Q N_{f}(u)+\frac{1}{T} \sum_{i=1}^{k} B_{i}\right]
$$

where $N_{f}(u)$ is defined by (12). Denote

$$
\begin{aligned}
& \Theta_{\lambda}: P C^{1} \rightarrow P C^{1}, \quad u \longmapsto(I-Q) N(u, \lambda)-\frac{\lambda}{T} \sum_{i=1}^{k} B_{i}, \\
& K_{\lambda}(u)(t)=F\left\{\varphi^{-1}\left[t,\left(\rho+\lambda \sum_{t_{i}<t} B_{i}+F\left(\Theta_{\lambda}(u)\right)\right)\right]\right\}(t), \quad \forall t \in J,
\end{aligned}
$$

where $\rho=\rho(\lambda, u)$ is the solution of $\lambda \sum_{i=1}^{k} A_{i}+K_{\lambda}(u)=0$.

Obviously

$$
\Theta_{\lambda}(u)=(I-Q) \lambda N_{f}(u)-\frac{\lambda}{T} \sum_{i=1}^{k} B_{i}, \quad \text { and } \quad \Theta_{0}(\cdot)=0 .
$$

From $\left(1^{0}\right)$, we can see that $(I-Q) \lambda N_{f}(u) \in E\left(0, \frac{1}{12}\right) \subset L_{m}^{1}, \forall u \in \bar{\Omega}, \forall \lambda \in[0,1]$. Combining $\left(\mathrm{H}_{1}\right)$ and Lemma 2.2, we can see that there exists only one $\rho=\rho(\lambda, u) \in \mathcal{F}_{2}$ such that

$$
\sup _{t \in J}\left|\rho+\lambda \sum_{t_{i}<t} B_{i}+F\left(\Theta_{\lambda}(u)\right)\right|<1 \quad \text { and } \quad \lambda \sum_{i=1}^{k} A_{i}+K_{\lambda}(u)=0, \quad \forall \lambda \in[0,1], \forall u \in \bar{\Omega} .
$$

Let

$$
\begin{aligned}
& \Phi_{f}(u, \lambda):=P u+\lambda \sum_{t_{i}<t} A_{i}+\lambda \frac{1}{T} \sum_{i=1}^{k} B_{i}+Q N(u, \lambda)+K_{\lambda}(u) \\
&=P u+\lambda \sum_{t_{i}<t} A_{i}+\frac{1}{T} \sum_{i=1}^{k} B_{i}+Q N_{f}(u)+K_{\lambda}(u),
\end{aligned}
$$


then the fixed point of $\Phi_{f}(u, 1)$ is a solution for (1)-(4). Also problem (20) can be written in the equivalent form

$$
u=\Phi_{f}(u, \lambda)
$$

Since $f$ is Caratheodory, it is easy to see that $N(\cdot, \cdot)$ is continuous and sends bounded sets into equi-integrable sets. According to Lemma 2.3, we can conclude that $\Phi_{f}$ is compact continuous on $\bar{\Omega} \times[0,1]$. From Lemma 2.4, we can see that the problem (1)-(4) is equivalent to $u=\Phi_{f}(u, 1)$. We assume that for $\lambda=1,(21)$ does not have a solution on $\partial \Omega$, otherwise we complete the proof. Now, from hypothesis $\left(2^{0}\right)$, it follows that (21) has no solution for $(u, \lambda) \in \partial \Omega \times(0,1]$. For $\lambda=0,(20)$ is equivalent to the following usual differential equation boundary value problem:

$$
\left\{\begin{array}{l}
-\left(\varphi\left(t, u^{\prime}\right)\right)^{\prime}=Q N_{f}(u)+\frac{1}{T} \sum_{i=1}^{k} B_{i}\left(\lim _{t \rightarrow t_{i}^{-}} u(t), \lim _{t \rightarrow t_{i}^{-}} u^{\prime}(t)\right), \quad t \in(0, T), \\
u(0)=u(T), \quad \text { and } \quad \varphi\left(0, u^{\prime}(0)\right)=\varphi\left(T, u^{\prime}(T)\right),
\end{array}\right.
$$

and if $u$ is a solution to this problem, we must have

$$
\int_{0}^{T} f\left(t, u(t), u^{\prime}(t)\right) d t+\sum_{i=1}^{k} B_{i}\left(\lim _{t \rightarrow t_{i}^{-}} u(t), \lim _{t \rightarrow t_{i}^{-}} u^{\prime}(t)\right)=0 .
$$

When $\lambda=0$, the problem is a usual differential equation boundary value problem. Hence $\varphi\left(t, u^{\prime}(t)\right) \equiv c$, where $c \in \mathcal{F}_{2}$ is a constant mapping. Since $u(0)=u(T)$, for any $i \in\{1, \ldots, N\}$, there exists $r_{0}^{i} \in(0, T)$, such that $\left(u^{i}\right)^{\prime}\left(r_{0}^{i}\right)=0$, hence $\left(u^{i}\right)^{\prime} \equiv 0$, and we have $u \equiv l \in \mathcal{F}_{2}$. Thus, by (22) we have

$$
\int_{0}^{T} f(r, l, 0) d r+\sum_{i=1}^{k} B_{i}(l, 0)=0
$$

which, together with hypothesis $\left(2^{0}\right)$, implies that $u=l \notin \partial \Omega$. Thus we have proved that (21) has no solution $(u, \lambda)$ on $\partial \Omega \times[0,1]$, and then we find that, for each $\lambda \in[0,1]$, the Leray-Schauder degree $d_{L S}\left[I-\Phi_{f}(\cdot, \lambda), \Omega, 0\right]$ is well defined. From the homotopy invariant property of that degree, we have

$$
d_{L S}\left[I-\Phi_{f}(\cdot, 1), \Omega, 0\right]=d_{L S}\left[I-\Phi_{f}(\cdot, 0), \Omega, 0\right] .
$$

When $\lambda=0$, we have $\Theta_{0}(\cdot)=0$, and $K_{0}(u)=0, \forall u \in P C^{1}$. Thus

$$
\Phi_{f}(u, 0)=P u+\frac{1}{T} \sum_{i=1}^{k} B_{i}+Q N_{f}(u)+K_{0}(u)=P u+\frac{1}{T} \sum_{i=1}^{k} B_{i}+Q N_{f}(u)
$$

and then all the solutions of $u-\Phi_{f}(u, 0)=0$ belong to $\mathcal{F}_{2}$. Thus

$$
u-\Phi_{f}(u, 0)=u-P u-\frac{1}{T} \sum_{i=1}^{k} B_{i}-Q N_{f}(u)=-\frac{1}{T} \sum_{i=1}^{k} B_{i}-Q N_{f}(u), \quad \text { on } \bar{\Omega} \cap \mathcal{F}_{2} .
$$


By the properties of the Leray-Schauder degree, we have

$$
d_{L S}\left[I-\Phi_{f}(\cdot, 0), \Omega, 0\right]=(-1)^{N} d_{B}\left[\omega, \Omega \cap \mathcal{F}_{2}, 0\right]
$$

where the function $\omega$ is defined in (18) and $d_{B}$ denotes the Brouwer degree. Since, by hypothesis $\left(4^{0}\right)$, this last degree is different from zero. Thus (1)-(4) has a solution. This completes the proof.

In the following, we will give an application of Theorem 2.5.

Assume:

$\left(\mathrm{H}_{2}\right) f(t, u, v)=\delta g(t, u, v)$, where $\delta$ is a positive parameter, and

$$
g(t, u, v)=\tau(t)\left(|u|^{q_{1}(t)-1} u+\mu(t)|v|^{q_{2}(t)}\right)+\gamma(t)
$$

where $q_{1}, q_{2} \in C(I, \mathbb{R})$, and $0 \leq q_{1}(t)<q_{2}(t), \forall t \in J$ or $0 \leq q_{2}(t)<q_{1}(t), \forall t \in J$.

$\left(\mathrm{H}_{3}\right) \mu=\left(\mu^{1}, \ldots, \mu^{N}\right) \in C\left(J, \mathbb{R}^{N}\right), \quad \tau \in C(J, \mathbb{R})$, and $\tau$ satisfies $\tau_{1} \leq \min _{t \in J}|\tau(t)| \leq$ $\max _{t \in J}|\tau(t)| \leq \tau_{2}$, where $\tau_{1}$ and $\tau_{2}$ are positive constants.

Obviously, there exists a positive constant $\varepsilon$ such that

$$
C_{\#}:=\min _{t \in J}\left(\frac{\sigma}{2 N}\left|\frac{\sigma}{2} \varepsilon\right|^{q_{1}(t)}-\|\mu\|_{0}|N \varepsilon|^{q_{2}(t)}\right)>0
$$

where $\sigma=\frac{4 T}{4 T+1}$.

$\left(\mathrm{H}_{4}\right) \quad \sum_{i=1}^{k}\left|A_{i}\right| \leq \frac{\sigma}{4} \varepsilon, \sum_{i=1}^{k}\left|B_{i}\right| \leq \min \left\{\frac{1}{4} T \delta \tau_{1} C_{\#}, \min _{t \in I} \frac{\frac{\varepsilon}{4 t+1}^{p(t)}}{2\left(1+|N \varepsilon|^{q(t) p(t)} \frac{1}{q(t)}\right.}\right\}$.

$\left(\mathrm{H}_{5}\right) A_{i}^{j}(x, y) y^{j} \geq 0, \forall x, y \in \mathbb{R}^{N}, i=1, \ldots, k, j=1, \ldots, N$, and $\sum_{i=1}^{k} B_{i}(l, 0)=0, \forall l \in \mathbb{R}^{N}$.

$\left(\mathrm{H}_{6}\right) \gamma=\left(\gamma^{1}, \ldots, \gamma^{N}\right) \in C\left(J, \mathbb{R}^{N}\right)$ satisfies $\left|\gamma^{i}\right|_{0}<\frac{1}{2} \tau_{1} C_{\#}$, for any $j=1, \ldots, N$.

\section{Denote}

$$
\Omega_{\varepsilon}=\left\{u \in P C^{1} \mid \max _{1 \leq j \leq N}\left(\left|u^{j}\right|_{0}+\left|\left(u^{j}\right)^{\prime}\right|_{0}\right)<\varepsilon\right\} .
$$

Obviously, $\Omega_{\varepsilon}$ is an open subset of $P C^{1}$.

Theorem 2.6 If $\left(\mathrm{H}_{1}\right)-\left(\mathrm{H}_{6}\right)$ are satisfied, then problem (1)-(4) has at least one solution on $\overline{\Omega_{\varepsilon}}$, when the positive parameter $\delta$ is small enough.

Proof We denote $N(\cdot, \cdot): P C^{1} \times[0,1] \rightarrow L^{1}$ defined by

$$
N(u, \lambda)=\lambda N_{f}(u)+(1-\lambda)\left[Q N_{f}(u)+\frac{1}{T} \sum_{i=1}^{k} B_{i}\right],
$$

where $N_{f}(u)$ is defined by (12). 
Let us consider the following problem:

$$
\begin{aligned}
u & =\Phi_{f}(u, \lambda):=P u+\lambda \sum_{t_{i}<t} A_{i}+\lambda \frac{1}{T} \sum_{i=1}^{k} B_{i}+Q N(u, \lambda)+K_{\lambda}(u) \\
& =P u+\lambda \sum_{t_{i}<t} A_{i}+\frac{1}{T} \sum_{i=1}^{k} B_{i}+Q N_{f}(u)+K_{\lambda}(u) .
\end{aligned}
$$

Obviously, $u$ is a solution of (1)-(4) if and only if $u$ is a solution of the abstract equation (23) when $\lambda=1$. We only need to prove that the conditions of Theorem 2.5 are satisfied.

$\left(1^{0}\right)$ When the positive parameter $\delta$ is small enough, for any $u \in \overline{\Omega_{\varepsilon}}$, we can see that the mapping $t \longmapsto \delta g\left(t, u, u^{\prime}\right)$ belongs to $\left\{u \in L^{1} \mid\|u\|_{L^{1}}<\frac{1}{24}\right\}$.

$\left(2^{0}\right)$ We shall prove that for each $\lambda \in(0,1)$ the problem

$$
\left\{\begin{array}{l}
\left(\varphi\left(t, u^{\prime}\right)\right)^{\prime}=\lambda f\left(t, u, u^{\prime}\right), \quad t \in(0, T), t \neq t_{i}, \\
\lim _{t \rightarrow t_{i}^{+}} u(t)-\lim _{t \rightarrow t_{i}^{-}} u(t)=\lambda A_{i}\left(\lim _{t \rightarrow t_{i}^{-}} u(t), \lim _{t \rightarrow t_{i}^{-}} u^{\prime}(t)\right), \quad i=1, \ldots, k, \\
\lim _{t \rightarrow t_{i}^{+}} \varphi\left(t, u^{\prime}(t)\right)-\lim _{t \rightarrow t_{i}^{-}} \varphi\left(t, u^{\prime}(t)\right) \\
\quad=\lambda B_{i}\left(\lim _{t \rightarrow t_{i}^{-}} u(t), \lim _{t \rightarrow t_{i}^{-}} u^{\prime}(t)\right), \quad i=1, \ldots, k \\
u(0)=u(T), \quad \text { and } \varphi\left(0, u^{\prime}(0)\right)=\varphi\left(T, u^{\prime}(T)\right)
\end{array}\right.
$$

has no solution on $\partial \Omega_{\varepsilon}$.

If it is false, then there exists a $\lambda \in(0,1)$, and $u \in \partial \Omega_{\varepsilon}$ is a solution of (23). We have

$$
\varphi\left(t, u^{\prime}\right)=\rho+\lambda \sum_{t_{i}<t} B_{i}+\int_{0}^{t} \lambda f\left(r, u, u^{\prime}\right) d r, \quad \forall t \in J^{\prime}
$$

From the boundary value condition (4), we have

$$
\int_{0}^{T} f\left(r, u, u^{\prime}\right) d r+\sum_{i=1}^{k} B_{i}=0
$$

Since $u \in \partial \Omega_{\varepsilon}$, there exists an $j \in\{1, \ldots, N\}$ such that $\left|u^{j}\right|_{0}+\left|\left(u^{j}\right)^{\prime}\right|_{0}=\varepsilon$.

(i) Suppose that $\left|u^{j}\right|_{0} \geq \sigma \varepsilon$, then $\left|\left(u^{j}\right)^{\prime}\right|_{0} \leq(1-\sigma) \varepsilon$. Let $0<r_{2}<r_{1}<T$, according to (2) and $\left(\mathrm{H}_{4}\right)$, we have

$$
\begin{aligned}
\left|u^{j}\left(r_{1}\right)-u^{j}\left(r_{2}\right)\right| & =\left|\int_{r_{2}}^{r_{1}}\left(u^{j}\right)^{\prime}(r) d r+\sum_{r_{2}<t_{i}<r_{1}} A_{i}\right| \\
& \leq \int_{0}^{T}\left|\left(u^{j}\right)^{\prime}(r)\right| d r+\sum_{r_{2}<t_{i}<r_{1}}\left|A_{i}\right| \\
& \leq \int_{0}^{T}(1-\sigma) \varepsilon d r+\sum_{i=1}^{k}\left|A_{i}\right| \leq \frac{\sigma}{4} \varepsilon+\frac{\sigma}{4} \varepsilon=\frac{\sigma}{2} \varepsilon .
\end{aligned}
$$

Since $\left|u^{j}\right|_{0} \geq \sigma \varepsilon$, we get $\left|u^{j}(t)\right| \geq \frac{\sigma}{2} \varepsilon$ for any $t \in J$. Obviously, $u^{j}$ has constant sign on $J$. 
The condition $\left(\mathrm{H}_{3}\right)$ means that $\tau$ has constant sign on $J$. Thus $\tau u^{j}$ also has constant sign on $J$. Assume that $\tau u^{j}$ is positive, then we have

$$
\begin{aligned}
& \int_{0}^{T} f^{j}\left(t, u, u^{\prime}\right) d t+\sum_{i=1}^{k} B_{i}^{j} \\
& \quad \geq \int_{0}^{T} \delta\left\{|\tau(t)|\left(|u(t)|^{q_{1}(t)-1}\left|u^{j}(t)\right|-\left|\mu^{j}\right|_{0}\left|u^{\prime}(t)\right|^{q_{2}(t)}\right)-\left|\gamma^{j}(t)\right|\right\} d t-\sum_{i=1}^{k}\left|B_{i}\right| \\
& \quad \geq \frac{1}{4} T \delta \tau_{1} C_{\#}>0 .
\end{aligned}
$$

This is a contradiction to (24).

Assume that $\tau u^{j}$ is negative, from $\left(\mathrm{H}_{4}\right)$ and $\left(\mathrm{H}_{6}\right)$, then we have

$$
\begin{aligned}
& \int_{0}^{T} f^{j}\left(t, u, u^{\prime}\right) d t+\sum_{i=1}^{k} B_{i}^{j} \\
& \quad \leq \int_{0}^{T}-\delta\left\{|\tau(t)|\left(|u(t)|^{q_{1}(t)-1}\left|u^{j}(t)\right|-\left|\mu^{j}\right|_{0}\left|u^{\prime}(t)\right|^{q_{2}(t)}\right)-\left|\gamma^{j}(t)\right|\right\} d t+\sum_{i=1}^{k}\left|B_{i}\right| \\
& \quad \leq-\frac{1}{4} T \delta \tau_{1} C_{\#}<0 .
\end{aligned}
$$

This is a contradiction to (24).

(ii) Suppose that $\left|u^{j}\right|_{0}<\sigma \varepsilon$, then $(1-\sigma) \varepsilon<\left|\left(u^{j}\right)^{\prime}\right|_{0} \leq \varepsilon$. This implies that $\left|\left(u^{j}\right)^{\prime}\left(s_{2}\right)\right|>(1-$ $\sigma) \varepsilon=\frac{1}{4 T+1} \varepsilon$ for some $s_{2} \in J^{\prime}$. Without loss of generality, we may assume that $\left(u^{j}\right)^{\prime}\left(s_{2}\right)>0$, the discussion of the case of $\left(w^{j}\right)^{\prime}\left(s_{2}\right)<0$ is similar.

$\left(\mathrm{a}^{0}\right)$ Suppose that $\inf _{t \in J^{\prime}}\left(w^{j}\right)^{\prime}(t)>0$. From $\left(\mathrm{H}_{5}\right)$, we have

$$
A_{i}^{j}\left(\lim _{t \rightarrow t_{i}^{-}} u(t), \lim _{t \rightarrow t_{i}^{-}} u^{\prime}(t)\right) \geq 0, \quad i=1, \ldots, k
$$

Since

$$
u^{j}(t)=u^{j}(0)+\int_{0}^{t}\left(u^{j}\right)^{\prime}(t) d t+\sum_{t_{i}<t} A_{i}^{j}
$$

we can see that $u^{j}(t)$ is increasing, and $u^{j}(T)>u^{j}(0)$. This is a contradiction to (4).

$\left(\mathrm{b}^{0}\right)$ Suppose that $\inf _{t \in J^{\prime}}\left(u^{j}\right)^{\prime}(t)<0$. Then there exists $r_{0}^{j} \in J^{\prime}$ such that $\left(u^{j}\right)^{\prime}\left(r_{0}^{j}\right)<0$. Denote

$$
\varphi^{j}\left(t, u^{\prime}\right)(t)=\frac{\left|u^{\prime}\right|^{p(t)-1}\left(u^{j}\right)^{\prime}(t)}{\left(1+\left|u^{\prime}\right| q^{q(t) p(t)}\right)^{\frac{1}{q(t)}}} .
$$

We have

$$
\varphi^{j}\left(s_{2}, u^{\prime}\right)\left(s_{2}\right)-\varphi^{j}\left(r_{0}^{j}, u^{\prime}\right)\left(r_{0}^{j}\right)=\lambda \int_{r_{0}^{j}}^{s_{2}} f^{j}\left(t, u, u^{\prime}\right) d t+\lambda \sum_{r_{0}^{j}<t_{i}<s_{2}} B_{i}^{j}+\lambda \sum_{s_{2}<t_{i}<r_{0}^{j}} B_{i}^{j} .
$$


According to $\left(\mathrm{H}_{2}\right)$ and $\left(\mathrm{H}_{4}\right)$, when the positive parameter $\delta$ is small enough, we have

$$
\begin{aligned}
\frac{\left|\frac{\varepsilon}{4 T+1}\right|^{p\left(s_{2}\right)}}{\left(1+|N \varepsilon|^{q\left(s_{2}\right) p\left(s_{2}\right)} \frac{1}{q\left(s_{2}\right)}\right.} & \leq\left|\varphi^{j}\left(s_{2}, u^{\prime}\right)\left(s_{2}\right)\right| \leq\left|\varphi^{j}\left(s_{2}, u^{\prime}\right)\left(s_{2}\right)-\varphi^{j}\left(t_{0}^{j}, u^{\prime}\right)\left(t_{0}^{j}\right)\right| \\
& \leq \lambda \int_{0}^{T}\left|f^{j}\left(t, u, u^{\prime}\right)\right| d t+\lambda\left|\sum_{r_{0}^{j}<t_{i}<s_{2}} B_{i}^{j}\right|+\lambda\left|\sum_{s_{2}<t_{i}<r_{0}^{j}} B_{i}^{j}\right| \\
& \leq \delta \int_{0}^{T} \tau_{2}\left[|N \varepsilon|^{q_{1}(r)}+\|\mu\|_{0}|N \varepsilon|^{q_{2}(t)}+\|\gamma\|_{0}\right] d t+\sum_{i=1}^{k}\left|B_{i}\right| \\
& <\frac{\left.\left|\frac{\varepsilon}{4 T+1}\right|\right|^{p\left(s_{2}\right)}}{\left(1+|N \varepsilon|^{q\left(s_{2}\right) p\left(s_{2}\right)}\right)^{\frac{1}{q\left(s_{2}\right)}}} .
\end{aligned}
$$

This is a contradiction to (4).

$\left(\mathrm{c}^{0}\right)$ Suppose that $\inf _{t \in J^{\prime}}\left(u^{j}\right)^{\prime}(t)=0$. Similar to the proof of $\left(\mathrm{b}^{0}\right)$, we can get a contradiction to (4).

Summarizing this argument, for each $\lambda \in(0,1)$, problem (23) has no solution on $\partial \Omega_{\varepsilon}$.

$\left(3^{0}\right)$ From $\left(\mathrm{H}_{2}\right),\left(\mathrm{H}_{5}\right)$, and $\left(\mathrm{H}_{6}\right)$, it is easy to see that

$$
\omega(l)=\int_{0}^{T} f(t, l, 0) d t+\sum_{i=1}^{k} B_{i}(l, 0)=\int_{0}^{T}\left(\tau(t)|l|^{q_{1}(t)-1} l+\gamma(t)\right) d t=0
$$

has no solution on $\partial \Omega \cap \mathcal{F}_{2}$.

$\left(4^{0}\right)$ Let

$$
h(t, l, \lambda)=\lambda \omega(l)+(1-\lambda) \frac{\tau(t)}{|\tau(t)|} l=\lambda \int_{0}^{T}\left(\tau(t)|l|^{q_{1}(t)-1} l+\gamma(t)\right) d t+(1-\lambda) \frac{\tau(t)}{|\tau(t)|} l .
$$

According to $\left(\mathrm{H}_{6}\right)$, it is easy to see that $\omega^{i}(l)$ and $\frac{\tau(t)}{|\tau(t)|} l^{i}$ have the same sign for any $\left|l^{i}\right|=\varepsilon$. Denote

$$
\Psi(l, \lambda)=\int_{0}^{T} h(t, l, \lambda) d t
$$

For any $\lambda \in[0,1]$, we see that $\Psi(l, \lambda)=0$ does not have solutions on $\partial \Omega_{\varepsilon} \cap \mathcal{F}_{2}$, then the Brouwer degree

$$
d_{B}\left[\omega, \Omega_{\varepsilon} \cap \mathcal{F}_{2}, 0\right]=d_{B}\left[\Psi(l, 1), \Omega_{\varepsilon} \cap \mathcal{F}_{2}, 0\right]=d_{B}\left[\Psi(l, 0), \Omega_{\varepsilon} \cap \mathcal{F}_{2}, 0\right] \neq 0 .
$$

Since (23) does not have solutions on $\partial \Omega_{\varepsilon}$, we have

$$
d_{L S}\left[I-\Phi_{f}(\cdot, 1), \Omega_{\varepsilon}, 0\right]=d_{L S}\left[I-\Phi_{f}(\cdot, 0), \Omega_{\varepsilon}, 0\right] .
$$

Since

$$
d_{L S}\left[I-\Phi_{f}(\cdot, 0), \Omega_{\varepsilon}, 0\right]=(-1)^{N} d_{B}\left[\varpi, \Omega_{\varepsilon} \cap \mathcal{F}_{2}, 0\right]=(-1)^{N} d_{B}\left[\Psi(l, 0), \Omega_{\varepsilon} \cap \mathcal{F}_{2}, 0\right] \neq 0,
$$


we have

$$
d_{L S}\left[I-\Phi_{f}(\cdot, 1), \Omega_{\varepsilon}, 0\right]=(-1)^{N} d_{B}\left[\varpi, \Omega_{\varepsilon} \cap \mathcal{F}_{2}, 0\right] \neq 0 .
$$

This completes the proof.

\section{Dirichlet boundary value problems}

In this section, we will discuss the existence of solutions of variable exponent mean curvature impulsive system Dirichlet boundary value problems, i.e. the existence of a solution of (1)-(3) and (5).

\subsection{Preliminary}

Let us now consider the following simple problem:

$$
\left(\varphi\left(t, u^{\prime}(t)\right)\right)^{\prime}=g(t), \quad t \in(0, T), t \neq t_{i}
$$

with the following impulsive boundary value conditions:

$$
\left\{\begin{array}{l}
\lim _{t \rightarrow t_{i}^{+}} u(t)-\lim _{t \rightarrow t_{i}^{-}} u(t)=a_{i}, \quad i=1, \ldots, k, \\
\lim _{t \rightarrow t_{i}^{+}} \varphi\left(t, u^{\prime}(t)\right)-\lim _{t \rightarrow t_{i}^{-}} \varphi\left(t, u^{\prime}(t)\right)=b_{i}, \quad i=1, \ldots, k \\
u(0)=u(T)=0,
\end{array}\right.
$$

where $a_{i}, b_{i} \in \mathbb{R}^{N}, \sum_{i=1}^{k}\left|b_{i}\right|<1 ; g \in L^{1}$.

If $u$ is a solution of (25) with (26), by integrating (25) from 0 to $t$, we find that

$$
\varphi\left(t, u^{\prime}(t)\right)=\varphi\left(0, u^{\prime}(0)\right)+\sum_{t_{i}<t} b_{i}+\int_{0}^{t} g(t) d t, \quad \forall t \in J^{\prime}
$$

Denote $\rho=\varphi\left(0, u^{\prime}(0)\right)$.

From the definition of $\varphi$, we can see that

$$
\sup _{t \in J}\left|\rho+\sum_{t_{i}<t} b_{i}+F(g)(t)\right|<1 .
$$

Denote

$$
\widetilde{U}_{b}=\left\{h \in L^{1} \mid \text { there exists } \rho \in \mathbb{R}^{N} \text { such that } \sup _{t \in J}\left|\rho+\sum_{t_{i}<t} b_{i}+F(h)(t)\right|<1\right\} .
$$

By (27), we have

$$
u(t)=\sum_{t_{i}<t} a_{i}+F\left\{\varphi^{-1}\left[t,\left(\rho+\sum_{t_{i}<t} b_{i}+F(g)\right)\right]\right\}(t), \quad \forall t \in J .
$$

The boundary value conditions imply that

$$
\sum_{i=1}^{k} a_{i}+\int_{0}^{T} \varphi^{-1}\left\{t,\left[\rho+\sum_{t_{i}<t} b_{i}+F(g)(t)\right]\right\} d t=0 .
$$


Denote $a=\left(a_{1}, \ldots, a_{k}\right) \in \mathbb{R}^{k N}, b=\left(b_{1}, \ldots, b_{k}\right) \in \mathbb{R}^{k N}$. It is easy to see that $\rho$ is dependent on $a, b$, and $g$. For fixed $g \in \widetilde{U}_{b}, a \in \mathbb{R}^{k N}$, we define

$$
\Lambda_{(a, b, g)}(\rho)=\sum_{i=1}^{k} a_{i}+\int_{0}^{T} \varphi^{-1}\left\{t,\left[\rho+\sum_{t_{i}<t} b_{i}+F(g)(t)\right]\right\} d t .
$$

Denote

$$
\begin{aligned}
U_{a, b}= & \left\{h \in \widetilde{U}_{b} \mid \text { there exists } \rho \in \mathbb{R}^{N}\right. \text { such that } \\
& \left.\sup _{t \in J}\left|\rho+\sum_{t_{i}<t} b_{i}+F(h)(t)\right|<1 \text { and } \Lambda_{(a, b, h)}(\rho)=0\right\} .
\end{aligned}
$$

If (25) with (26) has a solution in $P C^{1}$, we must have $g(\cdot) \in U_{a, b}$.

Denote $W=\mathbb{R}^{2 k N} \times L^{1}$ with the norm

$$
\|w\|_{W}=\sum_{i=1}^{k}\left|a_{i}\right|+\sum_{i=1}^{k}\left|b_{i}\right|+\|g\|_{L^{1}}, \quad \forall w=(a, b, g) \in W,
$$

then $W$ is a Banach space.

Copying the proof of Lemma 2.2, we have the following.

Lemma 3.1 The function $\Lambda_{w}(\cdot)$ has the following properties:

$\left(1^{0}\right)$ For any fixed $(a, b) \in \mathcal{M}_{1} \times \mathcal{M}_{2}, \forall h \in U_{a, b}$, the equation

$$
\Lambda_{w}(\rho)=0, \quad \text { where } w=(a, b, h) \in W,
$$

has a unique solution $\rho(w) \in \mathbb{R}^{N}$, where $\mathcal{M}_{1}, \mathcal{M}_{2}$ are defined in (10) and (11).

$\left(2^{0}\right)$ For any fixed $a=\left(a_{1}, \ldots, a_{k}\right) \in \mathcal{M}_{1}, b=\left(b_{1}, \ldots, b_{k}\right) \in \mathcal{M}_{2}, U_{a, b}$ contains the open ball $E_{\#}\left(0, \frac{1}{12}\right):=\left\{u \in L^{1} \mid\|u\|_{L^{1}}<\frac{1}{12}\right\}$, and then defines a mapping $\rho: \mathcal{M}_{1} \times \mathcal{M}_{2} \times$ $E_{\#}\left(0, \frac{1}{12}\right) \rightarrow \mathbb{R}^{N}$.

$\left(3^{0}\right)$ The function $\rho: \mathcal{M}_{1} \times \mathcal{M}_{2} \times E_{\#}\left(0, \frac{1}{12}\right) \rightarrow \mathbb{R}^{N}$, defined in $\left(2^{0}\right)$, is continuous and bounded.

$\left(4^{0}\right)$ For any fixed $a=\left(a_{1}, \ldots, a_{k}\right) \in \mathcal{M}_{1}, b=\left(b_{1}, \ldots, b_{k}\right) \in \mathcal{M}_{2}$, the set $U_{a, b}$ is open and unbounded in $L^{1}$.

We continue now with our argument previous to Lemma 3.1.

Define the nonlinear operator $K_{(a, b)}: U_{a, b} \rightarrow P C^{1}$, as

$$
K_{(a, b)}(h)(t)=F\left\{\varphi^{-1}\left[t,\left(\rho+\sum_{t_{i}<t} b_{i}+F(h)\right)\right]\right\}(t), \quad \forall t \in J .
$$

Similar to the proof of the Lemma 2.3, we have the following.

Lemma 3.2 If $(a, b) \in \mathcal{M}_{1} \times \mathcal{M}_{2}$, then the operator $K_{(a, b)}(\cdot)$ is continuous and sends closed equi-integrable subsets of $U_{a, b}$ into relatively compact sets in $P C^{1}$. 
Denote

$$
G(u)=F\left\{\varphi^{-1}\left[t,\left(\rho+\sum_{t_{i}<t} B_{i}+F\left(N_{f}\right)(u)\right)\right]\right\}(t),
$$

and

$$
\Lambda_{u}^{*}(\rho)=\sum_{i=1}^{k} A_{i}+\int_{0}^{T} \varphi^{-1}\left\{t,\left[\rho+\sum_{t_{i}<t} B_{i}+\int_{0}^{t} f\left(r, u, u^{\prime}\right) d r\right]\right\} d t
$$

where $A_{i}, B_{i}$ are defined in (19), $\rho$ is a solution of $\Lambda_{u}^{*}(\rho)=0$.

Similar to the proof of Lemma 2.4, we have the following.

Lemma 3.3 If $\left(\mathrm{H}_{1}\right)$ is satisfied, then $u$ is a solution of (1)-(3) and (5), if and only if $u$ is a solution of the following abstract operator equation:

$$
u=\sum_{t_{i}<t} A_{i}+G(u)
$$

\subsection{Existence of solutions}

In this subsection, we will apply the Leray-Schauder degree to deal with the existence of solutions of (1)-(3) and (5).

Theorem 3.4 If $\left(\mathrm{H}_{1}\right)$ is satisfied, $\Omega \subset P C^{1}$ is open bounded such that the following conditions hold:

$\left(1^{0}\right)$ For any $u \in \bar{\Omega}$, the mapping $t \rightarrow f\left(t, u, u^{\prime}\right)$ belongs to $\left\{u \in L^{1} \mid\|u\|_{L^{1}}<\frac{1}{12}\right\}$.

$\left(2^{0}\right)$ For each $\lambda \in(0,1)$, the problem

$$
\left\{\begin{array}{l}
\left(\varphi\left(t, u^{\prime}\right)\right)^{\prime}=\lambda f\left(t, u, u^{\prime}\right), \quad t \in(0, T), t \neq t_{i}, \\
\quad \lim _{t \rightarrow t_{i}^{+}} u(t)-\lim _{t \rightarrow t_{i}^{-}} u(t) \\
\quad=\lambda A_{i}\left(\lim _{t \rightarrow t_{i}^{-}} u(t), \lim _{t \rightarrow t_{i}^{-}} u^{\prime}(t)\right), \quad i=1, \ldots, k, \\
\quad \lim _{t \rightarrow t_{i}^{+}} \varphi\left(t, u^{\prime}(t)\right)-\lim _{t \rightarrow t_{i}^{-}} \varphi\left(t, u^{\prime}(t)\right) \\
\quad=\lambda B_{i}\left(\lim _{t \rightarrow t_{i}^{-}} u(t), \lim _{t \rightarrow t_{i}^{-}} u^{\prime}(t)\right), \quad i=1, \ldots, k, \\
u(0)=u(T)=0
\end{array}\right.
$$

has no solution on $\partial \Omega$;

$\left(3^{0}\right) \quad 0 \in \Omega$

Then (1)-(3), and (5) have a solution on $\bar{\Omega}$.

Proof Denote

$$
G_{\lambda}(u)(t)=F\left\{\varphi^{-1}\left[t,\left(\rho+\lambda \sum_{t_{i}<t} B_{i}+F\left(\lambda N_{f}(u)\right)\right)\right]\right\}(t), \quad \forall t \in J,
$$

where $\rho=\rho(\lambda, u)$ is the solution of $\lambda \sum_{i=1}^{k} A_{i}+G_{\lambda}(u)=0, N_{f}(u)$ is defined by (12), $A_{i}, B_{i}$ are defined in (19). 
From $\left(1^{0}\right)$, we can see that $\lambda N_{f}(u) \in E_{\#}\left(0, \frac{1}{12}\right) \subset L^{1}, \forall u \in \bar{\Omega}, \forall \lambda \in[0,1]$. Combining $\left(\mathrm{H}_{1}\right)$ and Lemma 3.1, we can see that there exists only one $\rho=\rho(\lambda, u) \in \mathcal{F}_{2}$ such that

$$
\sup _{t \in J}\left|\rho+\lambda \sum_{t_{i}<t} B_{i}+F\left(\lambda N_{f}(u)\right)\right|<1 \quad \text { and } \quad \lambda \sum_{i=1}^{k} A_{i}+G_{\lambda}(u)=0, \quad \forall \lambda \in[0,1], \forall u \in \bar{\Omega} .
$$

Let

$$
\Psi_{f}(u, \lambda):=\lambda \sum_{t_{i}<t} A_{i}+G_{\lambda}(u)
$$

then the fixed point of $\Psi_{f}(u, 1)$ is a solution for (1)-(3) and (5). Also problem (28) can be written in the equivalent form

$$
u=\Psi_{f}(u, \lambda)
$$

Since $f$ is Caratheodory, it is easy to see that $N(\cdot)$ is continuous and sends bounded sets into equi-integrable sets. According to Lemma 3.2, we can conclude that $\Psi_{f}$ is compact continuous on $\bar{\Omega} \times[0,1]$. From Lemma 3.3, we can see that problem (1)-(3) and (5) is equivalent to $u=\Psi_{f}(u, 1)$. We assume that for $\lambda=1$, (29) does not have a solution on $\partial \Omega$, otherwise we complete the proof. Now, from hypothesis $\left(2^{0}\right)$, it follows that (29) has no solution for $(u, \lambda) \in \partial \Omega \times(0,1]$. For $\lambda=0,(28)$ is equivalent to the following usual differential equation boundary value problem:

$$
\left\{\begin{array}{l}
-\left(\varphi\left(t, u^{\prime}\right)\right)^{\prime}=0, \quad t \in(0, T) \\
u(0)=u(T)=0
\end{array}\right.
$$

When $\lambda=0$, the problem is a usual differential equation boundary value problem. Hence $\varphi\left(t, u^{\prime}(t)\right) \equiv c$, where $c \in \mathcal{F}_{2}$ is a constant mapping. Since $u(0)=u(T)$, for any $i \in\{1, \ldots, N\}$, there exists $r_{0}^{i} \in(0, T)$, such that $\left(u^{i}\right)^{\prime}\left(r_{0}^{i}\right)=0$, hence $\left(u^{i}\right)^{\prime} \equiv 0$, we have $u \equiv l \in \mathcal{F}_{2}$. Thus, we have

$$
u=0 \text {, }
$$

which, together with hypothesis $\left(3^{0}\right)$, implies that $0 \in \Omega$. Thus we have proved that (29) has no solution $(u, \lambda)$ on $\partial \Omega \times[0,1]$, then we see that, for each $\lambda \in[0,1]$, the Leray-Schauder degree $d_{L S}\left[I-\Psi_{f}(\cdot, \lambda), \Omega, 0\right]$ is well defined. From the condition $\left(3^{0}\right)$ and the homotopy invariant property of that degree, we have

$$
d_{L S}\left[I-\Psi_{f}(\cdot, 1), \Omega, 0\right]=d_{L S}\left[I-\Psi_{f}(\cdot, 0), \Omega, 0\right]=1 \neq 0 .
$$

Thus (1)-(3) and (5) has a solution. This completes the proof.

In the following, we will give an application of Theorem 3.4.

Assume the following.

$\left(\mathrm{H}_{7}\right) f(t, u, v)=\delta g(t, u, v)$, where $\delta$ is a positive parameter, $g$ is defined in $\left(\mathrm{H}_{2}\right)$. 
Denote

$$
\Omega_{*}=\left\{u \in P C^{1}\left|\|u\|_{1}<2 N(1+T) \max _{t \in J}\right| \varphi^{-1}\left(t, \frac{11}{12}\right) \mid\right\} .
$$

Theorem 3.5 If $\left(\mathrm{H}_{1}\right)$ and $\left(\mathrm{H}_{7}\right)$ are satisfied, then problem (1)-(3) and (5) has at least one solution on $\overline{\Omega_{*}}$, when the positive parameter $\delta$ is small enough.

Proof Let us consider the following problem:

$$
u=\Psi_{f}(u, \lambda):=\lambda \sum_{t_{i}<t} A_{i}+G_{\lambda}(u)
$$

Obviously, $u$ is a solution of (1)-(3) and (5) if and only if $u$ is a solution of the abstract equation (30) when $\lambda=1$. We only need to prove that the conditions of Lemma 3.1 are satisfied.

$\left(1^{0}\right)$ When the positive parameter $\delta$ is small enough, for any $u \in \overline{\Omega_{*}}$, we can see that the mapping $t \longmapsto \delta g\left(t, u, u^{\prime}\right)$ belongs to $\left\{u \in L^{1} \mid\|u\|_{L^{1}}<\frac{1}{12}\right\}$.

$\left(2^{0}\right)$ We shall prove that for each $\lambda \in(0,1)$ the problem

$$
\left\{\begin{array}{l}
\left(\varphi\left(t, u^{\prime}\right)\right)^{\prime}=\lambda f\left(t, u, u^{\prime}\right), \quad t \in(0, T), t \neq t_{i}, \\
\lim _{t \rightarrow t_{i}^{+}} u(t)-\lim _{t \rightarrow t_{i}^{-}} u(t)=\lambda A_{i}\left(\lim _{t \rightarrow t_{i}^{-}} u(t), \lim _{t \rightarrow t_{i}^{-}} u^{\prime}(t)\right), \quad i=1, \ldots, k, \\
\lim _{t \rightarrow t_{i}^{+}} \varphi\left(t, u^{\prime}(t)\right)-\lim _{t \rightarrow t_{i}^{-}} \varphi\left(t, u^{\prime}(t)\right) \\
\quad=\lambda B_{i}\left(\lim _{t \rightarrow t_{i}^{-}} u(t), \lim _{t \rightarrow t_{i}^{-}} u^{\prime}(t)\right), \quad i=1, \ldots, k, \\
u(0)=u(T)=0
\end{array}\right.
$$

has no solution on $\partial \Omega_{*}$.

If it is false, then there exists a $\lambda \in(0,1)$, and $u \in \partial \Omega_{*}$ is a solution of (30). We have

$$
\varphi\left(t, u^{\prime}\right)=\rho+\lambda \sum_{t_{i}<t} B_{i}+\int_{0}^{t} \lambda f\left(r, u, u^{\prime}\right) d r, \quad \forall t \in J^{\prime}
$$

From the proof of Lemma 2.2, we can see that $|\rho|<\frac{2}{3}$. Obviously

$$
\left|\rho+\lambda \sum_{t_{i}<t} B_{i}+\int_{0}^{t} \lambda f\left(r, u, u^{\prime}\right) d r\right|<\frac{11}{12}
$$

It is easy to verify that $\|u\|_{1}<2 N(1+T) \max _{t \in J}\left|\varphi^{-1}\left(t, \frac{11}{12}\right)\right|$.

This is a contradiction.

Thus, for each $\lambda \in(0,1)$, problem (30) has no solution on $\partial \Omega_{*}$.

$\left(3^{0}\right)$ Obviously $0 \in \Omega_{*}$.

This completes the proof.

\section{Neumann boundary value problems}

In this section, we will discuss the existence of solutions of variable exponent mean curvature impulsive system Neumann boundary value problems, i.e. the existence of a solution of (1)-(3) and (6). 


\subsection{Preliminary}

Let us now consider the following simple problem:

$$
\left(\varphi\left(t, u^{\prime}(t)\right)\right)^{\prime}=g(t), \quad t \in(0, T), t \neq t_{i}
$$

with the following impulsive boundary value conditions:

$$
\left\{\begin{array}{l}
\lim _{t \rightarrow t_{i}^{+}} u(t)-\lim _{t \rightarrow t_{i}^{-}} u\left(t_{i}\right)=a_{i}, \quad i=1, \ldots, k, \\
\lim _{t \rightarrow t_{i}^{+}} \varphi\left(t, u^{\prime}(t)\right)-\lim _{t \rightarrow t_{i}^{-}} \varphi\left(t, u^{\prime}(t)\right)=b_{i}, \quad i=1, \ldots, k, \\
u^{\prime}(0)=u^{\prime}(T)=0,
\end{array}\right.
$$

where $a_{i}, b_{i} \in \mathbb{R}^{N}, \sum_{i=1}^{k}\left|b_{i}\right|<1 ; g \in L^{1}$ and it satisfies $\int_{0}^{T} g(t) d t+\sum_{i=1}^{k} b_{i}=0$.

Denote $a=\left(a_{1}, \ldots, a_{k}\right) \in \mathbb{R}^{k N}, b=\left(b_{1}, \ldots, b_{k}\right) \in \mathbb{R}^{k N}$, and

$$
L_{m}^{1}=\left\{v \in L^{1} \mid \int_{0}^{T} v(t) d t=0\right\} .
$$

Let $h(t)=g(t)+\frac{1}{T} \sum_{i=1}^{k} b_{i}$, then $h \in L_{m}^{1} . u^{\prime}(0)=u^{\prime}(T)=0$ implies $\varphi\left(0, u^{\prime}(0)\right)=\varphi(T$, $\left.u^{\prime}(T)\right)=0$.

If $u$ is a solution of (31) with (32), by integrating (31) from 0 to $t$, we find that

$$
\varphi\left(t, u^{\prime}(t)\right)=\sum_{t_{i}<t} b_{i}+\int_{0}^{t}\left[h(r)-\frac{1}{T} \sum_{i=1}^{k} b_{i}\right] d r, \quad \forall t \in J^{\prime} .
$$

From the definition of $\varphi$, we can see that

$$
\sup _{t \in J}\left|\varphi\left(t, u^{\prime}(t)\right)\right|=\sup _{t \in J}\left|\sum_{t_{i}<t} b_{i}+F\left(h-\frac{1}{T} \sum_{i=1}^{k} b_{i}\right)(t)\right|<1 .
$$

Denote

$$
V_{b}=\left\{h \in L_{m}^{1}\left|\sup _{t \in J}\right| \sum_{t_{i}<t} b_{i}+F\left(h-\frac{1}{T} \sum_{i=1}^{k} b_{i}\right)(t) \mid<1\right\} .
$$

By (33), we have

$$
u(t)=u(0)+\sum_{t_{i}<t} a_{i}+F\left\{\varphi^{-1}\left[t,\left(\sum_{t_{i}<t} b_{i}+F\left(h-\frac{1}{T} \sum_{i=1}^{k} b_{i}\right)\right)\right]\right\}(t), \quad \forall t \in J .
$$

We can split $L^{1}$ as $L^{1}=L_{m}^{1}+\mathscr{F}_{2}$, where $\mathcal{F}_{2}$ is the $N$-dimensional subspace of constant mappings. The operator $Q$ is a continuous projection from $L^{1}$ onto $\mathcal{F}_{2}$. Let us consider the subset $\widehat{V}_{b}$ of $L^{1}$ which is given by

$$
\widehat{V}_{b}=V_{b}+\mathcal{F}_{2},
$$

and the nonlinear operator $M_{*}: \widehat{V}_{b} \rightarrow P C^{1}$, as

$$
M_{*}(h)(t)=F\left\{\varphi^{-1}\left[t,\left(\sum_{t_{i}<t} b_{i}+F(h)\right)\right]\right\}(t), \quad \forall t \in J .
$$


Copying the proof of Lemma 2.3, we have the following.

Lemma 4.1 The operator $\left(M_{*} \circ \Theta_{b}\right)(\cdot)$ is continuous and sends closed equi-integrable subsets of $\widehat{V}_{b}$ into relatively compact sets in $P C^{1}$.

Denote

$$
\begin{aligned}
& \Theta_{\#}\left(N_{f}(u)\right)=(I-Q) N_{f}(u)-\frac{1}{T} \sum_{i=1}^{k} B_{i}, \\
& M(u)=F\left\{\varphi^{-1}\left[t,\left(\sum_{t_{i}<t} B_{i}+F\left(\Theta_{\#}\left(N_{f}(u)\right)\right)\right)\right]\right\},
\end{aligned}
$$

where $B_{i}$ is defined in (19).

Similar to the proof of Lemma 2.4, we have the following.

Lemma 4.2 $u$ is a solution of (1)-(3) and (6) if and only if $u$ is a solution of the following abstract equation:

$$
u=P u+\sum_{t_{i}<t} A_{i}+\frac{1}{T} \sum_{i=1}^{k} B_{i}+Q N_{f}(u)+M(u),
$$

where $A_{i}, B_{i}$ are defined in (19).

\subsection{Existence of solutions}

In this subsection, we will apply the Leray-Schauder degree to deal with the existence of solutions for (1)-(3) and (6).

Assume the following.

$\left(\mathrm{H}_{8}\right) \quad \sum_{i=1}^{k}\left|B_{i}\right| \leq \frac{1}{3}$.

Theorem 4.3 If $\left(\mathrm{H}_{8}\right)$ is satisfied, $\Omega$ is an open bounded set in $P C^{1}$ such that the following conditions hold:

$\left(1^{0}\right)$ For any $u \in \bar{\Omega}$, the mapping $t \rightarrow f\left(t, u, u^{\prime}\right)$ belongs to $\left\{u \in L^{1} \mid\|u\|_{L^{1}}<\frac{1}{6}\right\}$.

$\left(2^{0}\right)$ For each $\lambda \in(0,1)$, the problem

$$
\left\{\begin{array}{l}
\left(\varphi\left(t, u^{\prime}\right)\right)^{\prime}=\lambda f\left(t, u, u^{\prime}\right), \quad t \in(0, T), t \neq t_{i}, \\
\lim _{t \rightarrow t_{i}^{+}} u(t)-\lim _{t \rightarrow t_{i}^{-}} u(t)=\lambda A_{i}\left(\lim _{t \rightarrow t_{i}^{-}} u(t), \lim _{t \rightarrow t_{i}^{-}} u^{\prime}(t)\right), \quad i=1, \ldots, k, \\
\lim _{t \rightarrow t_{i}^{+}} \varphi\left(t, u^{\prime}(t)\right)-\lim _{t \rightarrow t_{i}^{-}} \varphi\left(t, u^{\prime}(t)\right) \\
\quad=\lambda B_{i}\left(\lim _{t \rightarrow t_{i}^{-}} u(t), \lim _{t \rightarrow t_{i}^{-}} u^{\prime}(t)\right), \quad i=1, \ldots, k, \\
u^{\prime}(0)=u^{\prime}(T)=0
\end{array}\right.
$$

has no solution on $\partial \Omega$.

$\left(3^{0}\right)$ The equation

$$
\omega(l):=\frac{1}{T} \int_{0}^{T} f(r, l, 0) d r+\frac{1}{T} \sum_{i=1}^{k} B_{i}(l, 0)=0
$$

has no solution on $\partial \Omega \cap \mathcal{F}_{2}$; 
$\left(4^{0}\right)$ The Brouwer degree $d_{B}\left[\omega, \Omega \cap \mathcal{F}_{2}, 0\right] \neq 0$.

Then (1)-(3) and (6) has a solution on $\bar{\Omega}$.

Proof It is similar to the proof of Theorem 2.6, we omit it here.

In the following, we will give an application of Theorem 4.3.

Assume the following.

$\left(\mathrm{H}_{9}\right) \quad \sum_{i=1}^{k} B_{i}(l, 0)=0, \forall l \in \mathbb{R}^{N}$.

$\left(\mathrm{H}_{10}\right) \gamma=\left(\gamma^{1}, \ldots, \gamma^{N}\right) \in C\left(J, \mathbb{R}^{N}\right)$ satisfies $\left|\gamma^{i}\right|_{0}<\frac{1}{2} \tau_{1} C_{\#}$, for any $j=1, \ldots, N$, where $C_{\#}$ is defined before $\left(\mathrm{H}_{4}\right)$.

Denote

$$
\Omega_{\varepsilon}=\left\{u \in P C^{1} \mid \max _{1 \leq j \leq N}\left(\left|u^{j}\right|_{0}+\left|\left(u^{j}\right)^{\prime}\right|_{0}\right)<\varepsilon\right\} .
$$

Obviously, $\Omega_{\varepsilon}$ is an open subset of $P C^{1}$.

Theorem 4.4 If $\left(\mathrm{H}_{2}\right)-\left(\mathrm{H}_{4}\right)$ and $\left(\mathrm{H}_{8}\right)-\left(\mathrm{H}_{10}\right)$ are satisfied, then problem (1)-(3) and (6) has at least one solution on $\overline{\Omega_{\varepsilon}}$, when the positive parameter $\delta$ is small enough.

Proof We denote $N(\cdot, \cdot): P C^{1} \times[0,1] \rightarrow L^{1}$ defined by

$$
N(u, \lambda)=\lambda N_{f}(u)+(1-\lambda)\left[Q N_{f}(u)+\frac{1}{T} \sum_{i=1}^{k} B_{i}\right] .
$$

Let us consider the problem

$$
\begin{aligned}
u & =\Phi_{f}(u, \lambda)=P u+\lambda \sum_{t_{i}<t} A_{i}+\lambda \frac{1}{T} \sum_{i=1}^{k} B_{i}+Q N(u, \lambda)+M_{\lambda}(u) \\
& =P u+\lambda \sum_{t_{i}<t} A_{i}+\frac{1}{T} \sum_{i=1}^{k} B_{i}+Q N_{f}(u)+M_{\lambda}(u),
\end{aligned}
$$

where $A_{i}, B_{i}$ are defined in (19).

It is easy to see $u$ is a solution of (1)-(3) and (6) if and only if $u$ is a solution of the abstract equation (34) when $\lambda=1$. We only need to prove that the conditions of Theorem 4.3 are satisfied.

$\left(1^{0}\right)$ When the positive parameter $\delta$ is small enough, for any $u \in \overline{\Omega_{\varepsilon}}$, we can see that the mapping $t \longmapsto \delta g\left(t, u, u^{\prime}\right)$ belongs to $\left\{u \in L^{1} \mid\|u\|_{L^{1}}<\frac{1}{6}\right\}$.

$\left(2^{0}\right)$ We shall prove that for each $\lambda \in(0,1)$ the problem

$$
\left\{\begin{array}{l}
\left(\varphi\left(t, u^{\prime}\right)\right)^{\prime}=\lambda f\left(t, u, u^{\prime}\right), \quad t \in(0, T), t \neq t_{i}, \\
\quad \lim _{t \rightarrow t_{i}^{+}} u(t)-\lim _{t \rightarrow t_{i}^{-}} u(t) \\
\quad=\lambda A_{i}\left(\lim _{t \rightarrow t_{i}^{-}} u(t), \lim _{t \rightarrow t_{i}^{-}} u^{\prime}(t)\right), \quad i=1, \ldots, k, \\
\lim _{t \rightarrow t_{i}^{+}} \varphi\left(t, u^{\prime}(t)\right)-\lim _{t \rightarrow t_{i}^{-}} \varphi\left(t, u^{\prime}(t)\right) \\
\quad=\lambda B_{i}\left(\lim _{t \rightarrow t_{i}^{-}} u(t), \lim _{t \rightarrow t_{i}^{-}} u^{\prime}(t)\right), \quad i=1, \ldots, k, \\
u^{\prime}(0)=u^{\prime}(T)=0,
\end{array}\right.
$$

has no solution on $\partial \Omega_{\varepsilon}$, i.e. (34) has no solution on $\partial \Omega_{\varepsilon}$. 
If it is false, then there exists a $\lambda \in(0,1)$, and $u \in \partial \Omega_{\varepsilon}$ is a solution of (34). Integrating (35) from 0 to $t$, we have

$$
\varphi\left(t, u^{\prime}\right)=\lambda \sum_{t_{i}<t} B_{i}+\int_{0}^{t} \lambda f\left(t, u, u^{\prime}\right) d t, \quad \forall t \in J^{\prime}
$$

From the boundary value condition (6), we have

$$
\int_{0}^{T} f\left(t, u, u^{\prime}\right) d t+\sum_{i=1}^{k} B_{i}=0
$$

Since $u \in \partial \Omega_{\varepsilon}$, there exists an $j \in\{1, \ldots, N\}$ such that $\left|u^{j}\right|_{0}+\left|\left(u^{j}\right)^{\prime}\right|_{0}=\varepsilon$.

(i) Suppose that $\left|u^{j}\right|_{0} \geq \sigma \varepsilon,(1-\sigma) \varepsilon \leq\left|\left(u^{j}\right)^{\prime}\right|_{0} \leq \varepsilon$.

Similar to the proof of (i) of $\left(2^{0}\right)$ of Theorem 2.6, we get a contradiction to (36).

(ii) Suppose that $\left|u^{j}\right|_{0}<\sigma \varepsilon,(1-\sigma) \varepsilon<\left|\left(u^{j}\right)^{\prime}\right|_{0} \leq \varepsilon$. This implies that $\left|\left(u^{j}\right)^{\prime}\left(r_{*}\right)\right|>(1-\sigma) \varepsilon=$ $\frac{1}{4 T+1} \varepsilon$ for some $r_{*} \in J$.

Denote

$$
\varphi^{j}\left(t, u^{\prime}\right)(t)=\frac{\left|u^{\prime}\right|^{p(t)-1}\left(u^{j}\right)^{\prime}(t)}{\left(1+\left|u^{\prime}\right|^{q(t) p(t)}\right)^{\frac{1}{q(t)}}} .
$$

Since $u^{\prime}(0)=u^{\prime}(T)=0$, we have

$$
\varphi^{j}\left(r_{*}, u^{\prime}\right)\left(r_{*}\right):=\frac{\left|u^{\prime}\right|^{p\left(r_{*}\right)-1}\left(u^{j}\right)^{\prime}\left(r_{*}\right)}{\left(1+\left|u^{\prime}\right|^{q\left(r_{*}\right) p\left(r_{*}\right)}\right)^{\frac{1}{q\left(r_{*}\right)}}}=\lambda \int_{0}^{r_{*}} f^{j}\left(r, u, u^{\prime}\right) d r+\lambda \sum_{0<t_{i}<r_{*}} B_{i}^{j} .
$$

According to $\left(\mathrm{H}_{10}\right)$, when the positive parameter $\delta$ is small enough, we have

$$
\begin{aligned}
\frac{\left|\frac{\varepsilon}{4 T+1}\right|^{p\left(r_{*}\right)}}{\left(1+|N \varepsilon|^{q\left(r_{*}\right) p\left(r_{*}\right)} \frac{1}{q\left(r_{*}\right)}\right.} & \leq\left|\varphi^{j}\left(r_{*}, u^{\prime}\right)\left(r_{*}\right)\right| \leq \lambda \int_{0}^{r_{*}}\left|f^{j}\left(t, u, u^{\prime}\right)\right| d t+\lambda\left|\sum_{0<t_{i}<r_{*}} B_{i}^{j}\right| \\
& \leq \delta \int_{0}^{T} \tau_{2}\left[|N \varepsilon|^{q 1(t)}+\|\mu\|_{0}|N \varepsilon|^{q_{2}(t)}+\|\gamma\|_{0}\right] d t+\sum_{i=1}^{k}\left|B_{i}\right| \\
& <\frac{\left|\frac{\varepsilon}{4 T+1}\right|^{p\left(r_{*}\right)}}{\left(1+|N \varepsilon|^{q\left(r_{*}\right) p\left(r_{*}\right)}\right)^{\frac{1}{q\left(r_{*}\right)}}} .
\end{aligned}
$$

This is a contradiction.

Summarizing this argument, for each $\lambda \in(0,1)$, problem (34) has no solution on $\partial \Omega_{\varepsilon}$. $\left(3^{0}\right)$ and $\left(4^{0}\right)$ similar to the proof of $\left(3^{0}\right)$ and $\left(4^{0}\right)$ of Theorem 2.6.

\section{Competing interests}

The authors declare that they have no competing interests.

\section{Authors' contributions}

All authors typed, read, and approved the final manuscript.

\section{Author details}

${ }^{1}$ College of Information and Management Science, Henan Agricultural University, Zhengzhou, Henan 450002, China. ${ }^{2}$ College of Mathematics and Information Science, Zhengzhou University of Light Industry, Zhengzhou, Henan 450002, China. 


\section{Acknowledgements}

The authors are highly grateful to the referees for careful reading and comments on this paper. The research is partly supported by the National Natural Science Foundation of China (11326161) and the key projects of Science and Technology Research of the Henan Education Department (14A110011).

\section{Received: 18 March 2014 Accepted: 25 May 2014 Published online: 23 September 2014}

\section{References}

1. Bainov, D, Simeonov, P: Systems with Impulse Effect. Ellis Horwood, Chichester (1989)

2. Ding, W, Wang, Y: New result for a class of impulsive differential equation with integral boundary conditions. Commun. Nonlinear Sci. Numer. Simul. 18, 1095-1105 (2013)

3. Fu, X, Yan, B, Liu, Y: Introduction of Impulsive Differential Systems. Science Press, Beijing (2005)

4. Hernández, E, O'Regan, D: Existence results for a class of abstract impulsive differential equations. Bull. Aust. Math. Soc. $87,366-385$ (2013)

5. Jiao, JJ, Chen, LS, Li, LM: Asymptotic behavior of solutions of second-order nonlinear impulsive differential equations. J. Math. Anal. Appl. 337, 458-463 (2008)

6. Liang, RX: Existence of solutions for impulsive Dirichlet problems with the parameter inequality reverse. Math. Methods Appl. Sci. 36, 1929-1939 (2013)

7. Liu, LS, Hu, LL, Wu, YH: Positive solutions of two-point boundary value problems for systems of nonlinear second-order singular and impulsive differential equations. Nonlinear Anal. 69(11), 3774-3789 (2008)

8. Liu, XZ, Guo, DJ: Periodic boundary value problems for a class of second-order impulsive integro-differential equations in Banach spaces. J. Math. Anal. Appl. 216, 284-302 (1997)

9. Shen, JH, Wang, WB: Impulsive boundary value problems with nonlinear boundary conditions. Nonlinear Anal. 69(11), 4055-4062 (2008)

10. Wang, J, Yan, B: Global properties and multiple solutions for boundary-value problems of impulsive differential equations. Electron. J. Differ. Equ. 2013, Article ID 171 (2013)

11. Yang, T: Impulsive Systems and Control: Theory and Applications. Nova Science Publishers, Huntington (2001)

12. Yao, MP, Zhao, AM, Yan, JR: Periodic boundary value problems of second-order impulsive differential equations Nonlinear Anal. 70(1), 262-273 (2009)

13. Zavalishchin, ST, Sesekin, AN: Dynamic Impulse Systems: Theory and Applications. Kluwer Academic, Dordrecht (1997)

14. Bogun, I: Existence of weak solutions for impulsive $p$-Laplacian problem with superlinear impulses. Nonlinear Anal., Real World Appl. 13, 2701-2707 (2012)

15. Cabada, A, Tomeček, J: Extremal solutions for nonlinear functional $\phi$-Laplacian impulsive equations. Nonlinear Anal. 67, 827-841 (2007)

16. Dong, W, Xu, JF, Zhang, XY: Weak solutions for a $p$-Laplacian impulsive differential equation. Abstr. Appl. Anal. 2013, Article ID 106023 (2013)

17. $\mathrm{Xu}, J \mathrm{~J}, \mathrm{Wei}, \mathrm{ZL}$, Ding, YZ: Existence of weak solutions for $p$-Laplacian problem with impulsive effects. Taiwan. J. Math $17,501-515$ (2013)

18. Yang, Y, Meng, F: Existence of positive solution for impulsive boundary value problem with $p$-Laplacian in Banach spaces. Math. Methods Appl. Sci. 36, 650-658 (2013)

19. Zhang, QH, Qiu, ZM, Liu, XP: Existence of solutions for prescribed variable exponent mean curvature system boundary value problems. Nonlinear Anal. 71, 2964-2975 (2009)

20. Brubaker, ND, Lindsay, AE: The onset of multi-valued solutions of a prescribed mean curvature equation with singular non-linearity. Eur. J. Appl. Math. 24, 631-656 (2013)

21. Filippucci, R: Entire radial solutions of elliptic systems and inequalities of the mean curvature type. J. Math. Anal. Appl. 334(1), 604-620 (2007)

22. Greco, A: On the existence of large solutions for equations of prescribed mean curvature. Nonlinear Anal. 34, 571-583 (1998)

23. Ivochkina, NM: The Dirichlet problem for the equations of curvature of order $m$. Leningr. Math. J. 2, 631-654 (1991)

24. Lancaster, KE, Siegel, D: Existence and behavior of the radial limits of a bounded capillary surface at a corner. Pac. J. Math. 176(1), 165-194 (1996)

25. Pan, HJ: One-dimensional prescribed mean curvature equation with exponential nonlinearity. Nonlinear Anal. 70(2), 999-1010 (2009)

26. Pan, HJ, Xing, RX: Nonexistence of solutions for prescribed mean curvature equations on a ball. J. Math. Anal. Appl. 406, 482-501 (2013)

27. Takimoto, K: Solution to the boundary blowup problem for k-curvature equation. Calc. Var. Partial Differ. Equ. 26(3), 357-377 (2006)

28. Trudinger, NS: The Dirichlet problem for the prescribed curvature equations. Arch. Ration. Mech. Anal. 111, 153-179 (1990)

29. Zhi, G, Zhao, L, Chen, G, Liu, X, Zhang, Q: Existence of solutions and nonnegative solutions for prescribed variable exponent mean curvature impulsive system initialized boundary value problems. J. Inequal. Appl. 2010, Article ID $915739(2010)$

30. Acerbi, E, Mingione, G: Regularity results for a class of functionals with nonstandard growth. Arch. Ration. Mech. Anal. $156,121-140(2001)$

31. Chen, Y, Levine, S, Rao, M: Variable exponent, linear growth functionals in image restoration. SIAM J. Appl. Math. 66(4), 1383-1406 (2006)

32. Rủžička, M: Electrorheological Fluids: Modeling and Mathematical Theory. Lecture Notes in Mathematics, vol. 1784. Springer, Berlin (2000)

33. Zhikov, W: Averaging of functionals of the calculus of variations and elasticity theory. Math. USSR, Izv. 29, 33-36 (1987)

34. Allegretto, W: Form estimates for the $p(x)$-Laplacean. Proc. Am. Math. Soc. 135(7), 2177-2185 (2007) 
35. Dong, R, Guo, Y, Zhang, QH: Existence of solutions for weighted $p(t)$-Laplacian impulsive integro-differential system with integral boundary value conditions. Bull. Malays. Math. Soc. 36, 419-434 (2013)

36. Dong, R, Zhang, QH: Solutions and nonnegative solutions for a weighted variable exponent impulsive integro-differential system with multi-point and integral mixed boundary value problems. Bound. Value Probl. 2013, Article ID 161 (2013)

37. Fan, $\mathrm{XL}$ : Global $C^{1, \alpha}$ regularity for variable exponent elliptic equations in divergence form. J. Differ. Equ. 235, 397-417 (2007)

38. Fan, $X L$, Zhao, D: On the spaces $L^{p(x)}(\Omega)$ and $W^{m p(x)}(\Omega)$. J. Math. Anal. Appl. 263, 424-446 (2001)

39. Fan, XL, Zhang, QH, Zhao, D: Eigenvalues of $p(x)$-Laplacian Dirichlet problem. J. Math. Anal. Appl. 302, 306-317 (2005)

40. El Hamidi, A: Existence results to elliptic systems with nonstandard growth conditions. J. Math. Anal. Appl. 300, 30-42 (2004)

41. Kováčik, O, Rákosník, J: On the spaces $L^{p(x)}(\Omega)$ and $W^{k, p(x)}(\Omega)$. Czechoslov. Math. J. 41, 592-618 (1991)

42. Marcellini, P: Regularity and existence of solutions of elliptic equations with $(p, q)$-growth conditions. J. Differ. Equ. 90 1-30 (1991)

43. Mihăilescu, M, Rădulescu, V: Continuous spectrum for a class of nonhomogeneous differential operators. Manuscr. Math. 125, 157-167 (2008)

44. Musielak, J: Orlicz Spaces and Modular Spaces. Lecture Notes in Mathematics, vol. 1034. Springer, Berlin (1983)

45. Samko, SG: Densness of $C_{0}^{\infty}\left(\mathbb{R}^{N}\right)$ in the generalized Sobolev spaces $W^{m, p(x)}\left(\mathbb{R}^{N}\right)$. Dokl. Akad. Nauk, Ross. Akad. Nauk 369(4), 451-454 (1999)

46. Zhang, QH: Existence of positive solutions to a class of $p(x)$-Laplacian equations with singular nonlinearities. Appl. Math. Lett. 348, 38-50 (2008)

47. Zhang, QH: Existence, nonexistence and asymptotic behavior of boundary blow-up solutions to $p(x)$-Laplacian problems with singular coefficient. Nonlinear Anal. 71, 2964-2975 (2009)

48. Zhi, GZ, Guo, YR, Wang, Y, Zhang, QH: Existence of solutions and nonnegative solutions for a class of $p(t)$-Laplacian differential systems with multipoint and integral boundary value conditions. Bound. Value Probl. 2013, Article ID 106 (2013)

49. Hai, DD, Shivaji, R: An existence result on positive solutions of p-Laplacian systems. Nonlinear Anal. 56, 1007-1010 (2004)

50. Diening, L, Harjulehto, P, Hästö, P, Rǔžička, M: Lebesgue and Sobolev Spaces with Variable Exponents. Lecture Notes in Mathematics, vol. 2017. Springer, Berlin (2011)

51. Yoshida, N: Picone identities for half-linear elliptic operators with $p(x)$-Laplacians and applications to Sturmian comparison theory. Nonlinear Anal. 74, 5631-5642 (2011)

doi:10.1186/s13661-014-0139-x

Cite this article as: Yin et al.: On the prescribed variable exponent mean curvature impulsive system boundary value problems. Boundary Value Problems 2014 2014:139.

\section{Submit your manuscript to a SpringerOpen ${ }^{\ominus}$ journal and benefit from:}

- Convenient online submission

- Rigorous peer review

- Immediate publication on acceptance

- Open access: articles freely available online

- High visibility within the field

- Retaining the copyright to your article 